\title{
ESTUDO DO PERFIL QUÍMICO DE CERVEJAS BRASILEIRAS: UMA AVALIAÇÃO ENTRE AS BEBIDAS ARTESANAIS E INDUSTRIAIS
}

\author{
Dorval M. Coelho Neto ${ }^{a}$, Laysa L. P. F. Moreira ${ }^{a}$, Eustáquio V. R. de Castroa, Warley B. Souza ${ }^{a}$, Paulo R. Filgueirasa,

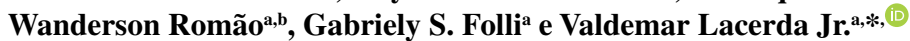

aUniversidade Federal do Espírito Santo, 29075-910 Vitória - ES, Brasil

'Instituto Federal do Espírito Santo, 29106-010 Vila Velha - ES, Brasil

Recebido em 22/08/2021; aceito em 30/11/2021; publicado na web em 27/01/2022

\begin{abstract}
STUDY OF THE CHEMICAL PROFILE OF BRAZILIAN BEERS: AN ASSESSMENT BETWEEN ARTISANAL AND INDUSTRIAL DRINKS. Beer is commonly produced with four inputs: water, malt, hops, yeast and, in some cases, brewing adjuncts. Industrial and artisanal processes essentially follow the same steps and may undergo minor changes due to the style of beer. Despite this, many consumers attribute higher quality to craft beer compared to industrial ones. In this study, we applied the analytical techniques of infrared (IR), hydrogen nuclear magnetic resonance ( ${ }^{1} \mathrm{H}$ NMR) and mass spectrometry (ESI $( \pm)$ FT-ICR MS) to discriminate craft beers from industrial ones (brands common to the Brazilian market). In addition, Partial Least Squares (PLS) and Support Vector Machine (SVR) regression were used to estimate some beer properties ( $\mathrm{pH}$, total acidity (meq $\mathrm{L}^{-1}$, color $(\mathrm{EBC})$, bitterness (IBU), alcohol content $(\% \mathrm{v} / \mathrm{v})$ and density $\left.\left(\mathrm{g} \cdot \mathrm{mL}^{-1}\right)\right)$. The use of the IR allowed the identification of vibrations attributed to chemical compounds common to beverages, such as water $\left(3320\right.$ and $\left.1640 \mathrm{~cm}^{-1}\right)$, carbohydrates $\left(1500 \mathrm{~cm}^{-1}\right)$ and ethanol $(1050-1040$ and 875-865 $\left.\mathrm{cm}^{-1}\right) .{ }^{1} \mathrm{H}$ NMR showed good applicability in identifying classes of organic compounds in the beverage, where signs attributed to alcohols, organic acids, carbohydrates and amino acids were observed. The ESI(+)FT-ICR MS allowed the identification of chemical compounds present in beverages, allowing the construction of a fingerprint of the beers. In addition, the application of chemometric tools enabled the prediction of physicochemical properties, presenting promising results in the prediction of alcohol content (RMSEC 0.2430 and RMSEP 0.3929) and bitterness (RMSEC 1.3022 and RMSEP 1.6008), and also in the classification regarding the manufacturing process (craft and industrial beer).
\end{abstract}

Keywords: chemometrics; regression; PLS; SVR; PCA.

\section{INTRODUÇÃO}

A cerveja é uma bebida fermentada de cereais produzida a partir de quatro ingredientes básicos: a água, malte, lúpulo, levedura e, em alguns casos, adjuntos cervejeiros. ${ }^{1}$ No Brasil, observa-se um aumento expressivo no número de cervejarias. Segundo dados do Ministério da Agricultura, Pecuária e Abastecimento - MAPA, o país encerrou o ano de 2020 com 1383 cervejarias autorizadas (Figura 1.A), concentradas, principalmente, nas regiões Sul e Sudeste (Figura 1.B). ${ }^{2}$ É importante destacar que, somente entre os anos de
2018 a 2020, o número de cervejarias teve aumento superior a 100\%, quando comparado a somatória de 2000 a 2017. Nesses anos, não foram registrados anúncios expressivos de novas plantas fabris pelas grandes cervejarias nacionais e multinacionais, responsáveis pela produção de cervejas de larga escala. ${ }^{3}$ Dessa forma, esse aumento está fortemente relacionado à expansão de cervejarias artesanais. ${ }^{4}$

Segundo a Associação Brasileira da Cerveja Artesanal (ABRACERVA), a cerveja artesanal é aquela especial, que preza pela qualidade, ligada a cervejarias independentes, registradas no MAPA, sem ligação com grandes grupos econômicos e que produz,

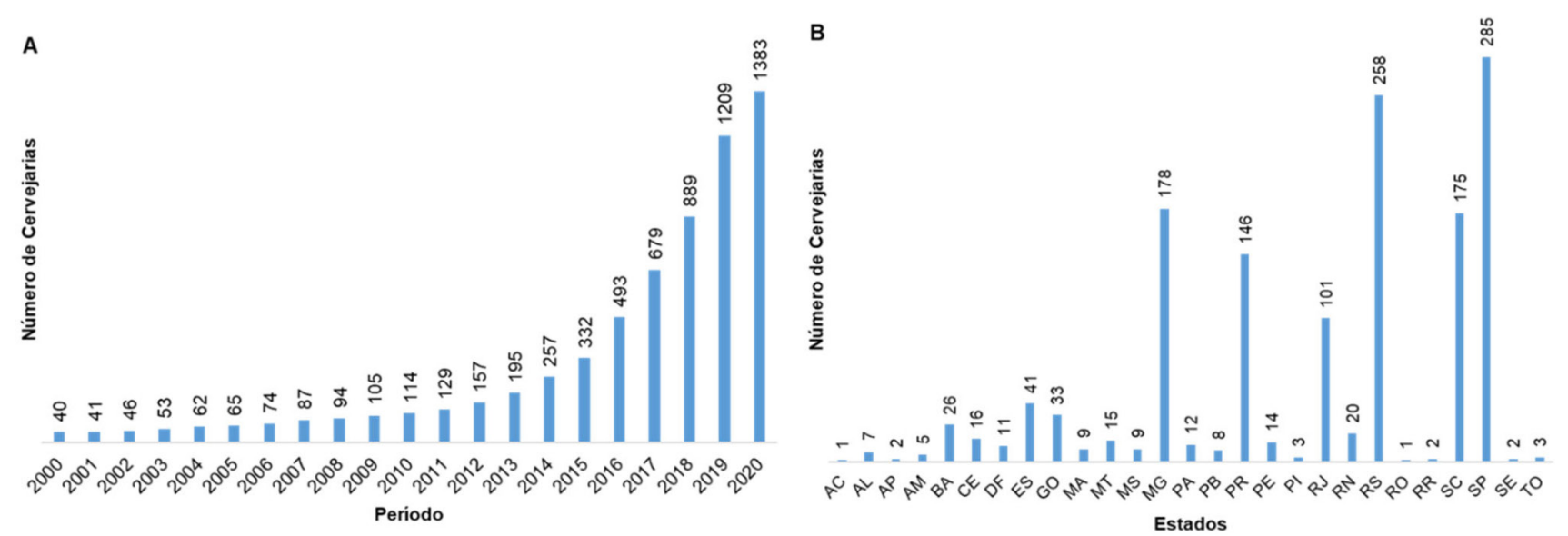

Figura 1. Evolução do número de cervejarias registradas no Brasil de 2000 a 2020 (A); Número de cervejarias registradas, por unidade da federação, em 2020 (B). Dados do Ministério da Agricultura, Pecuária e Abastecimento - MAPA ${ }^{2}$ 
no máximo, 416 mil litros/mês. ${ }^{5}$ Essa definição guarda alinhamento com a Brewers Association, associação norte americana de cervejeiros artesanais, que define a cerveja artesanal como aquela produzida por uma cervejaria independente, com menos de $25 \%$ da empresa sob propriedade de um membro da indústria de bebidas alcoólicas, com volume inferior a 714 milhões de litros/ano, cuja produção deve ser voltada a inovação, através da releitura de estilos clássicos e desenvolvimento de novos, mantendo foco na qualidade dos insumos tradicionais e no sabor no produto final. ${ }^{6} \mathrm{~A}$ produção em menor escala pode interferir no poder de negociação dos produtores que, combinado com processos que demandam maior tempo de execução, pode fazer com que o custo de produção da cerveja artesanal seja maior, quando comparado a cerveja industrial. Enquanto a cerveja industrial, é produzida em larga escala para alta distribuição. Essas cervejas, em geral, possuem processos e ingredientes que visam menor custo possível, resultado da combinação do maior poder de negociação devido a quantidade de insumos demandado e do tempo de produção que pode durar apenas um dia. Além disso, outra estratégia para redução dos custos está na substituição do malte de cevada por outros cereais de menor valor. ${ }^{7}$

A produção das cervejas industriais e artesanais, em geral, seguem procedimentos semelhantes, os quais podem ser descritos em sete etapas: moagem, mostura, fervura, fermentação, maturação, filtração e envase. A moagem do malte e, se for o caso, demais cereais, consiste na separação da casca e do endosperma do grão, permitindo seu melhor contato com a água. Na mostura, os cereais são colocados em água e submetido a um aquecimento gradual de 40 a $78^{\circ} \mathrm{C}$, permitindo a hidratação do grão e a ativação das enzimas ( $\alpha$-amilase e $\beta$-amilase) que converterão o amido do cereal em açúcares fermentáveis e solúveis em água. ${ }^{8}$ Posteriormente, o mosto (líquido açucarado) é submetido à fervura, etapa na qual será realizada a adição do lúpulo. A elevação na temperatura resulta na contração do anel dos $\alpha$-ácidos, resultando na formação de isômeros cis e trans, transformando-os em iso- $\alpha$-ácidos, responsáveis pelo amargor da bebida. ${ }^{9} \mathrm{Na}$ fervura também ocorrem reações de Maillard, na qual aminoácidos e carboidratos presentes no mosto reagem formando melanoidinas (cor amarelo âmbar), maltol (sabor de caramelo ou tostado) e funareol (sabor de morango), conforme representado na Figura $2 .^{10}$

Ao término da fervura, o mosto é resfriado para posterior inoculação e início da fermentação alcoólica. Nesse processo (Figura 3), a glicose é oxidada formando piruvato que por sua vez sofre descarboxilação, na forma de $\mathrm{CO}_{2}$, devido a ação da enzima piruvato descarboxilase. A presença da coenzima tiamina pirofosfato nesse sistema resulta na formação de acetaldeído, o qual sofre redução produzindo etanol, impactando no teor alcoólico final da bebida. ${ }^{11}$

A maturação procede a fermentação, consistindo na remoção das leveduras mortas e permitindo que as leveduras que ainda estão ativas no líquido catalisem a conversão de substâncias indesejadas em outras com sabores neutros, em adicional, a contínua produção

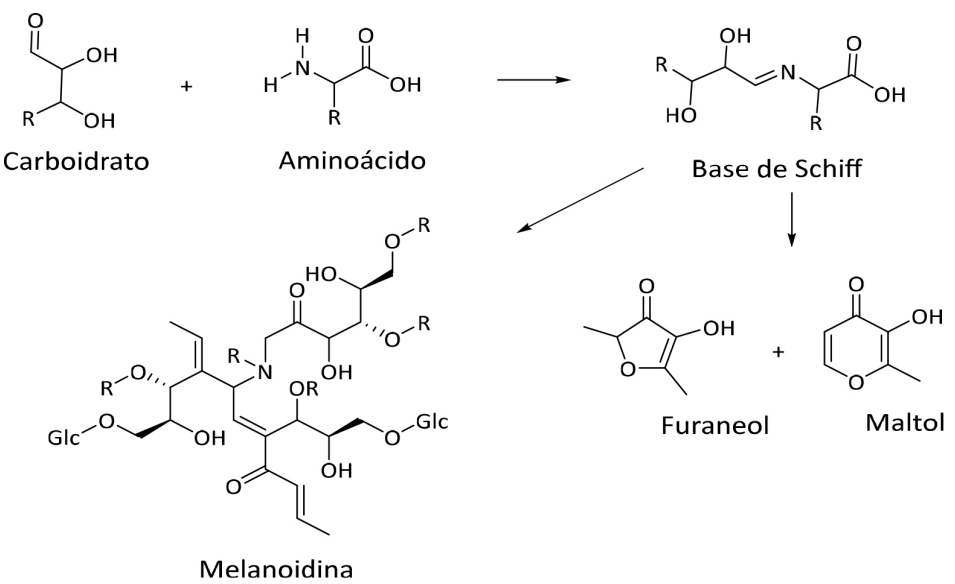

Figura 2. Mecanismo da reação de Maillard resultando na formação de melanoidinas, Furaneol e Maltol. Glc = Glicina e R=H, proteína, aminoácido ou Glicina ${ }^{10}$

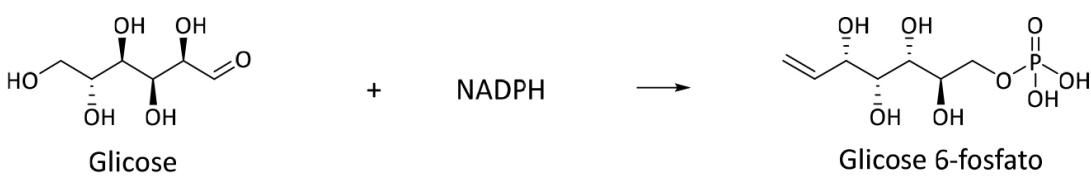

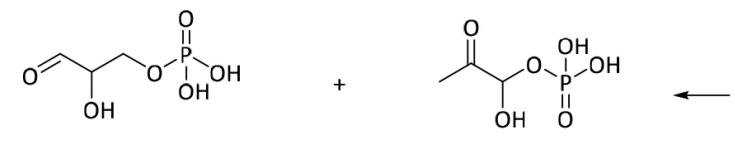

Gliceraldeído-3-fosfato

Fosfato de di-hidroxiacetona<smiles>O=C(COP(=O)(O)O)[C@@H](O)[C@H](O)[C@H](O)COP(=O)(O)O</smiles>

Frutose-1,6-bisfosfato

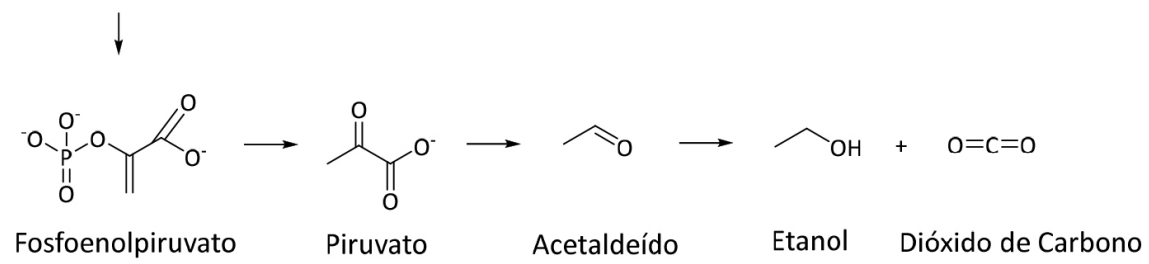

Figura 3. Mecanismo de conversão da glicose em etanol e dióxido de carbono pela ação das leveduras 
de $\mathrm{CO}_{2}$ auxilia na eliminação de compostos voláteis indesejáveis. Terminada a maturação a bebida poder ser submetida à filtração, objetivando a remoção de sólidos. ${ }^{1} \mathrm{O}$ envase, geralmente, é feito em recipientes metálicos, plásticos ou de vidro. No envase, também é feita a adição de gás carbônico $\left(\mathrm{CO}_{2}\right)$, denominada carbonatação. A carbonatação pode ser de maneira artificial por injeção ou natural por refementação. ${ }^{12}$ Após todo o processo produtivo, a bebida pode ser caracterizada quanto a cor (medido em EBC, do inglês European Brewery Convention), amargor (medido em IBU, do inglês International Bitterness Units), teor alcoólico (medido em \% $/ \mathrm{v}$ ), densidade (medido em $\mathrm{g} \cdot \mathrm{mL}^{-1}$ ), acidez total (medido em meq. $\mathrm{L}^{-1}$ ) e $\mathrm{pH}$, refletindo a contribuição das etapas descritas anteriormente nas propriedades da cerveja.

Apesar dos processos produtivos seguirem as mesmas etapas e, em muitos casos, ingredientes similares, existem diversos relatos de consumidores acerca da superioridade na qualidade, diversidade e sabor das cervejas artesanais frente as cervejas industriais. ${ }^{13}$ Muitos trabalhos tem abordado o emprego de técnicas analíticas, combinadas à quimiometria, voltadas a autenticação, classificação, monitoramento de processos produtivos e caracterização da bebida, adotando nessa abordagem a espectroscopia na região do infravermelho médio (MIR) e próximo (NIR), ${ }^{14-22}$ a ressonância magnética nuclear de hidrogênio $\left(\mathrm{RMN}\right.$ de $\left.{ }^{1} \mathrm{H}\right){ }^{23-31}$ e a espectrometria de massas (MS). ${ }^{32-35}$

No estudo de Giovenzana e colaboradores ${ }^{36}$ a técnica de NIR foi empregada para determinação de $\mathrm{pH}$, enquanto Mignani e colaboradores ${ }^{17}$ obtiveram sucesso com a utilização da técnica na validação de cervejas belgas. Ghasemi-Varnamkhasti e colaboradore ${ }^{20}$ utilizaram o NIR para monitorar o envelhecimento da bebida. Nos estudos de Almeida e colaboradores, ${ }^{23}$ Rodrigues e colaboradores ${ }^{26}$ e Petersen e colaboradores ${ }^{28}$ foi possível notar que o uso de RMN de ${ }^{1} \mathrm{H}$ tem boa aplicação na determinação da composição da bebida, em especial, na identificação de carboidratos. Entretanto, mais recentemente, no trabalho de Silva e colaboradores, ${ }^{29}$ foi apresentado o emprego desta técnica na discriminação de cervejas lager brasileiras. A aplicação de MS tem se mostrado promissora na identificação de falsificação de cervejas, conforme abordado no estudo de Pereira e colaboradores. ${ }^{34}$

Diante do exposto, o presente trabalho tem como objetivo avaliar o perfil químico de cervejas brasileiras artesanais e industriais mediante aplicação das técnicas analíticas de MIR, NIR, RMN de ${ }^{1} \mathrm{H}$ e ESI( \pm FT-ICR MS, visando a identificação de compostos químicos presentes nas cervejas industriais e artesanais por meio de modelos quimiométricos de regressão (Mínimos Quadrados Parciais, PLS, e Regressão por Vetores de Suporte, SVR) e análise por componentes principais, PCA. Adicionalmente, as bebidas avaliadas foram caracterizadas quanto as propriedades físico-químicas $(\mathrm{pH}$, cor, amargor, teor alcoólico e densidade).

\section{PARTE EXPERIMENTAL}

\section{Amostras}

Para a realização do estudo foram selecionadas um total de 30 amostras de cervejas. Dentre elas, ${ }^{21}$ cervejas industriais de rótulos comuns ao mercado brasileiro e 9 produzidas artesanalmente no escopo deste trabalho.

\section{Amostras industriais}

As amostras industriais foram adquiridas em supermercados da Grande Vitória/ES, comercializadas em latas de alumínio, contemplando 21 diferentes rótulos de 10 cervejarias distintas, sendo 10 puro malte e 11 com Adjuntos (Tabela 2). Nela, podemos observar que as amostras industrias selecionadas são, majoritariamente, cervejas do Estilo Pilsen e Lager com teor alcoólico entre 0,00 (L5 e P8) a 5,5\%v/v (R2). Além disso, é possível notar que os rótulos trazem poucas informações quanto as propriedades físico-químicas das bebidas, sendo o teor alcoólico a única propriedade informada em todas as embalagens. Dentre os rótulos analisados, somente a cerveja S10 traz informação sobre o amargor e a cor. A partir da avaliação da composição da bebida fornecida pelo fabricante, as cervejas foram classificadas em "Adjunto Industrial”, amostras produzidas com malte de cevada e adjuntos (cereais não maltados e carboidratos), e "Puro Malte Industrial", sendo aquelas fabricadas com malte de cevada e isenta de adjuntos.

\section{Amostras artesanais}

Com intuito de verificar possível distinção no perfil químico e nas propriedades físico-químicas das cervejas em função da composição e do processo fabril, foram produzidas 09 amostras de cervejas artesanais. Dentre elas, 1 puro malte (PPM) e 8 com adjuntos (P15M, P30M, P45M, P15A, P30A, P45A, PPM SC e P15M SC). Das amostras produzidas com adjuntos, 06 derivaram da substituição do malte de cevada, em 15, 30 e $45 \% \mathrm{~m} / \mathrm{m}$, por milho (P15M, P30M e P45M) e arroz (P15A, P30A e P45A) na etapa da brassagem e carbonatadas com $12 \mathrm{~g} \cdot \mathrm{L}^{-1}$ extrato de malte. Enquanto duas derivam na adição de sacarose $10 \mathrm{~g} \cdot \mathrm{L}^{-1}$ na etapa de carbonatação (PPM SC e P15M SC). A quantificação dos insumos usados na produção foi realizada no software BeerSmith, versão 2.3.12, ${ }^{37}$ conforme características apresentadas na Tabela 1 .

As etapas de mostura e fervura foram realizadas no equipamento BeerMax, o qual foi desenvolvido especialmente para as etapas quentes da produção cervejeira artesanal, possuindo sistema de aquecimento elétrico controlado por computador. As temperaturas empregadas são apresentadas na Figura 4. Para as etapas de fermentação e maturação (etapas frias) foi utilizado o freezer horizontal da marca Eletrolux,

Tabela 1. Perfil parametrizado, com os valores desejados, para a produção das cervejas artesanais puro malte e com adjuntos. Classificação com base na composição: Puro Malte Artesanal (PMA) e com Adjunto Artesanal (ADA)

\begin{tabular}{|c|c|c|c|c|c|}
\hline Cerveja & Estilo & Teor alcoólico $(\% \mathrm{v} / \mathrm{v})$ & Amargor (IBU) & Cor (EBC) & Classificação \\
\hline PPM & Pilsen & 4,8 & 10 & 12 & PMA \\
\hline $\mathrm{P} 15 \mathrm{M}$ & Pilsen & 4,8 & 10 & 12 & $\mathrm{ADA}$ \\
\hline $\mathrm{P} 30 \mathrm{M}$ & Pilsen & 4,8 & 10 & 12 & ADA \\
\hline $\mathrm{P} 45 \mathrm{M}$ & Pilsen & 4,8 & 10 & 12 & $\mathrm{ADA}$ \\
\hline $\mathrm{P} 15 \mathrm{~A}$ & Pilsen & 4,8 & 10 & 12 & ADA \\
\hline P30A & Pilsen & 4,8 & 10 & 12 & $\mathrm{ADA}$ \\
\hline $\mathrm{P} 45 \mathrm{~A}$ & Pilsen & 4,8 & 10 & 12 & $\mathrm{ADA}$ \\
\hline PPM SC & Pilsen & 4,8 & 10 & 12 & ADA \\
\hline P15M SC & Pilsen & 4,8 & 10 & 12 & ADA \\
\hline
\end{tabular}


modelo H500, acoplado a um controlador de temperatura marca Ageon, modelo G101.

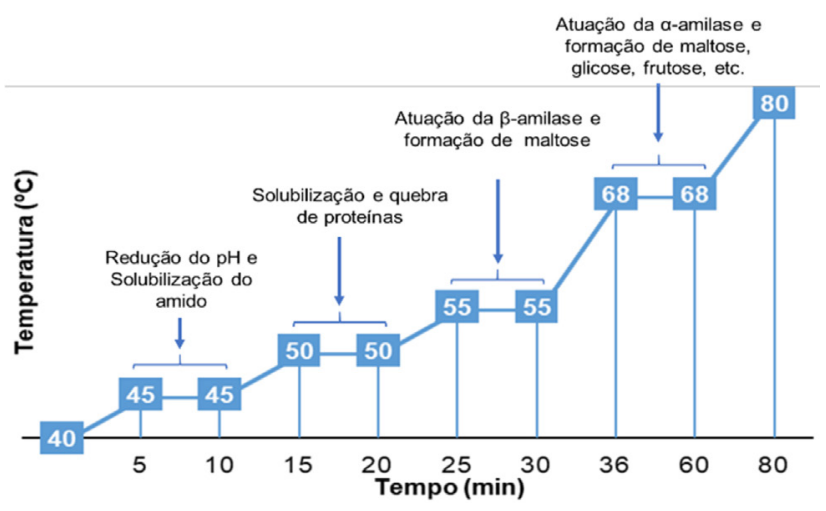

Figura 4. Rampa de temperatura aplicada durante a mostura ${ }^{10}$

A bebida foi mantida no fermentador a $(18 \pm 1)^{\circ} \mathrm{C}$ durante 15 dias. Após esse período, foi transferida para um maturador, a $(15 \pm 1){ }^{\circ} \mathrm{C}$ por 30 dias. A carbonatação da bebida ocorreu por refermentação, conforme descrito na Tabela 1 em garrafas de vidro âmbar de $600 \mathrm{~mL}$.

\section{Preparo da Amostra}

Como etapa preliminar a caracterização físico-química e obtenção dos dados instrumentais foi realizada a remoção dos gases característicos às cervejas, em especial o $\mathrm{CO}_{2}$. Para tal, $350 \mathrm{~mL}$ de cerveja foram transferidos para um béquer com capacidade de $1 \mathrm{~L}$ e posteriormente acondicionados em banho ultrassônico, fabricante LimpSonic, modelo LS-55DA-2/X, preenchido com água deionizada. As condições de otimização do banho de ultrassom foram: frequência de $40 \mathrm{kHz}$, potência $400 \mathrm{~W}$, tempo de cavitação de 6 minutos e temperatura do banho de $20{ }^{\circ} \mathrm{C} .{ }^{38}$ As amostras submetidas a esse processo foram denominadas amostras "desgaseificadas".

\section{Propriedades Físico-Químicas}

As propriedades físico-químicas das bebidas desgaseificadas foram determinadas de acordo com os métodos contidos no MOBV, ${ }^{39}$ quanto a densidade, grau alcoólico real, $\mathrm{pH}$ e acidez total.

A densidade do mosto, a $20^{\circ} \mathrm{C}$, foi medida através de densímetro eletrônico marca Anton Paar, modelo Density Meter DMA 35. O grau alcoólico foi determinado através da destilação da bebida e posterior medição da densidade do destilado a $20{ }^{\circ} \mathrm{C}$. O grau alcoólico real é expresso em \%v/v e está tabelado no MOBV de acordo com a densidade do destilado.

$\mathrm{O} \mathrm{pH}$ foi determinado por potenciometria a $20{ }^{\circ} \mathrm{C}$, usando pHmetro marca Metrohm, modelo $827 \mathrm{pH}$ lab, calibrado com solução Buffer, marca SpecSol, com pH $(7,00 \pm 0,02)$ a $(20,00 \pm 0,02){ }^{\circ} \mathrm{C}$ e depois outra solução da mesma marca com pH $(4,00 \pm 0,01)$ a $(20 \pm 0,02){ }^{\circ} \mathrm{C}$.

A acidez total foi determinada por titulação ácido-base, no qual $10 \mathrm{~mL}$ da amostra foi diluída em $100 \mathrm{~mL}$ de água deionizada e titulada com hidróxido de sódio $0,1 \mathrm{~N}$ em meio com 3 gotas de fenolftaleína. A concentração total de compostos ácidos na bebida foi calculada conforme a Equação 1, em que At representa a acidez total em meq. $\mathrm{L}^{-1}$, $\boldsymbol{n}$ o volume em $\mathrm{mL}$ de solução titulante gasto, $N$ a normalidade da solução de hidróxido de sódio e V o volume da amostra em mL.

$$
A t=\frac{1000 \cdot n \cdot N}{V}
$$

$\mathrm{O}$ amargor da bebida foi determinado em unidades internacionais de amargor (IBU, do inglês International Bitterness Units). O método mais utilizado para determinação dessa propriedade é o EBC. Ele consiste na acidificação de $10 \mathrm{~mL}$ de cerveja com $0,5 \mathrm{~mL}$ de ácido clorídrico $6 \mathrm{~mol} \mathrm{~L}^{-1}$ e com posterior adição de $20 \mathrm{~mL}$ de isooctano. Posteriormente a mistura foi agitada em agitador magnético por 15 minutos e centrifugada a 1000 rotações por minuto (rpm) durante 3 minutos. O sobrenadante (iso- $\alpha$-ácidos) foi removido e a absorbância no UV medida a $275 \mathrm{~nm}$, usando isooctano como branco. $\mathrm{O}$ amargor, em IBU, é determinado de acordo com a Equação 2, onde $A b s_{275 n m}$ é a absorbância. ${ }^{40}$

$$
I B U=A b s_{275 n m} \cdot 50
$$

A coloração foi determinada através do método $\mathrm{EBC} 9.6,{ }^{41}$ nele a cerveja desgaseificada é filtrada para retenção de partículas superiores a $45 \mu \mathrm{m}$ e, posteriormente, realizada a medição da absorbância da amostra a $430 \mathrm{~nm}$, por intermédio do equipamento de UV/VIS marca Perkin Elmer, modelo Lambda 45, e emprego da Equação 3, em que EBC representa a cor da bebida, $\mathrm{Abs}_{430 \mathrm{~nm}}$ a absorbância e $\boldsymbol{f}$ o fator de diluição da amostra.

$$
E B C=A b s_{430 n m} \cdot f \cdot 25
$$

\section{Análises Instrumentais}

\section{Espectroscopia na região do infravermelho}

As amostras desgaseificadas foram analisadas por infravermelho no equipamento Perkin Elmer, modelo Spectrum 400. Os espetros de NIR foram obtidos em 10000 a $4000 \mathrm{~cm}^{-1}$, com resolução de $8 \mathrm{~cm}^{-1}$ e 128 scans. Os espetros de MIR foram obtidos em 4000 a $650 \mathrm{~cm}^{-1}$, foram programados com resolução de $8 \mathrm{~cm}^{-1}$ e 32 scans. Vale destacar que as medidas foram realizadas em triplicata sendo avaliado o espectro médio resultante.

\section{Espectroscopia de Ressonância Magnética Nuclear}

As análises de $\mathrm{RMN}$ de ${ }^{1} \mathrm{H}$ foram realizadas no equipamento marca Varian, modelo VNMRS 400, operando com um campo magnético de 9,4 T, utilizando uma sonda de $5 \mathrm{~mm}$. Os espectros foram obtidos das amostras desgaseificadas de cerveja $(600 \mu \mathrm{L})$, a $26^{\circ} \mathrm{C}$, utilizando, como padrão externo em tubo de inserção, solução de ácido maleico (6,28 ppm) em água deuterada na concentração de $6,7 \mathrm{mg} \cdot \mathrm{mL}^{-1}$. Os parâmetros de aquisição foram definidos com frequência de 399,73 Mhz, janela espectral de $6410,30 \mathrm{~Hz}$, tempo de aquisição de 3,834 s, frequência de pré-saturação de 4,6092 Hz, tempo de espera (relaxação) de $2 \mathrm{~s}$, sequência de pulso PRESAT (supressão do sinal atribuído aos hidrogênios da água em 4,64 ppm), pulso de $90^{\circ}$ e 32 scans.

\section{$\operatorname{ESI}( \pm) F T-I C R M S$}

Os espectros de massas foram adquiridos em triplicata no espectrômetro de massas ESI(土)FT-ICR MS, marca Bruker Daltonics, Bremen, Alemanha, modelo 9.4 T Solarix. A calibração do equipamento foi feita a partir da solução de Arginina $(\mathrm{m} / \mathrm{z}, 200$ a 1500). No preparo da amostra, $20 \mu \mathrm{L}$ de cerveja foram diluídos em $1 \mathrm{~mL}$ de acetonitrila, observando a formação de precipitados, os quais foram filtrados para evitar possível entupimento do capilar do equipamento. A faixa de aquisição foi de $\mathrm{m} / \mathrm{z}, 150$ a 1500 , modos positivo e negativo, e precisão massa de $<2 \mathrm{ppm}$.

\section{Análise Quimiométrica}

Os dados obtidos por meio das técnicas analíticas descritas no tópico anterior foram tratados utilizando o software Matlab R2013a ${ }^{42}$ 
para criação dos modelos de regressão PLS e SVR e Análise por Componentes Principais (PCA). As amostras foram divididas, sendo $70 \%$ delas (21 amostras) para o grupo de calibração e $30 \%$ para o grupo de teste (9 amostras), de acordo com o algoritmo KennardStone. ${ }^{43}$ As amostras do grupo de calibração e teste foram as mesmas para todos os modelos.

Para $\mathrm{RMN}$ de ${ }^{1} \mathrm{H}$, o tratamento interval correlation shifting (icoshift $)^{44,45}$ foi usado para alinhamento dos espectros. Os prétratamentos utilizados para criação dos modelos quimiométricos foram o adaptive iteratively reweighed penalized least squares (airPLS), ${ }^{46}$ derivada de primeira ou segunda ordem com 3, 9, 7 ou 15 janelas e polinômio de segundo grau, autoescalar, centralização e normalização na média, standard normal variate (SNV), multiplicative scatter correlation (MSC), autoescalar, também combinações deles. O pré-tratamento com melhores resultados para predição de cada propriedade físico-química é apresentado na sessão de Resultados e Discussão.

A calibração cruzada para otimização do número de variáveis latentes (VL) das regressões por mínimos quadrados parciais (PLS) foi realizada por metodologia $\mathrm{k}$-fold,${ }^{47} \mathrm{com} \mathrm{k}$ igual a 5 . Também foram realizados modelos de regressões por máquina de vetores de suporte (SVR), no qual mapeia amostras em espaço de alta dimensão através da função Kernel e as amostras podem ser separadas pelo hiperplano de separação otimizado $(\mathrm{OSH}){ }^{48,49}$ Os parâmetros dos modelos SVR também foram otimizados (custo (C), margem ( $(\varepsilon)$ e gama $(\gamma)$ ). Os modelos SVR foram avaliados segundo os parâmetros de desempenho de acurácia (root mean square error of calibration [RMSEC] e root mean square error of prediction [RMSEP]) e de linearidade (coeficiente de determinação, $\mathrm{R}^{2}$ ). Já os modelos de PLS também foram avaliados segundo os parâmetros de acurácia, linearidade, limite de detecção (LD) e limite de quantificação (LQ).

\section{RESULTADOS E DISCUSSÃO}

\section{Propriedades Físico-Químicas}

Nesta seção apresentaremos os resultados obtidos, quanto as propriedades físico-químicas $(\mathrm{pH}$, cor, amargor, acidez total, teor alcoólico real e densidade), de todas as bebidas avaliadas no presente estudo (Tabela 2). Na Tabela 2 é possível observar que todas as cervejas apresentam caráter ácido, com $\mathrm{pH}$ variando entre 4,52 (I2) e 5,00 (Q9 e T8), valores que vão ao encontro com os relatados na literatura. ${ }^{50} \mathrm{Em}$ geral, as cervejas puro malte apresentam maiores valores quando comparadas as bebidas com adjuntos. Em relação a acidez total foram observados valores entre 18 (J7) a 40 meq $\mathrm{L}^{-1}$ (K6), condizentes com relatos da literatura. ${ }^{51}$

Tabela 2. Caracterização físico-química das amostras cervejas comerciais e artesanais. Desvio-padrão informado entre parêntesis. Dado não informado (N/I).

\begin{tabular}{|c|c|c|c|c|c|c|c|c|c|}
\hline Amostras & Estilo & Classificação $^{1}$ & $\mathrm{pH}$ & $\begin{array}{l}\text { Acidez total } \\
\left(\text { meq } \cdot \mathrm{L}^{-1}\right)\end{array}$ & Cor (EBC) & $\begin{array}{l}\text { Amargor } \\
\text { (IBU) }\end{array}$ & $\begin{array}{c}\text { Teor Alcoólico } \\
\text { Fab. }^{2}(\% \mathrm{v} / \mathrm{v})\end{array}$ & $\begin{array}{c}\text { Teor Alcoólico } \\
(\% \mathrm{v} / \mathrm{v})\end{array}$ & $\begin{array}{l}\text { Densidade } \\
\left(\mathrm{g} \mathrm{mL}^{-1}\right)\end{array}$ \\
\hline$\overline{\mathrm{A} 1}$ & $\mathrm{~N} / \mathrm{I}$ & PMI & $4,91(0,02)$ & $39,00(0,02)$ & $10,85(0,50)$ & $10,267(0,001)$ & 4,1 & $3,70(0,05)$ & $1,0066(0,0001)$ \\
\hline B2 & Pilsen & ADI & $4,77(0,01)$ & $30,00(0,02)$ & $7,25(0,10)$ & $9,450(0,001)$ & 4,6 & $4,10(0,05)$ & $1,0058(0,0001)$ \\
\hline $\mathrm{C} 3$ & Lager & PMI & $4,95(0,01)$ & $37,00(0,04)$ & $11,13(0,33)$ & $11,300(0,001)$ & 4,7 & $4,20(0,01)$ & $1,0074(0,0001)$ \\
\hline D1 & Pilsen & ADI & $4,55(0,01)$ & $29,00(0,10)$ & $8,90(0,01)$ & $10,016(0,001)$ & 4,7 & $4,50(0,08)$ & $1,0065(0,0001)$ \\
\hline E4 & Lager & PMI & $4,75(0,03)$ & $28,00(0,02)$ & $6,90(0,20)$ & $18,316(0,001)$ & 5,0 & $4,10(0,10)$ & $1,0047(0,0001)$ \\
\hline F5 & Pilsen & ADI & $4,67(0,02)$ & $25,00(0,40)$ & $7,53(0,10)$ & $9,933(0,001)$ & 4,5 & $4,20(0,10)$ & $1,0067(0,0001)$ \\
\hline G6 & Pilsen & ADI & $4,65(0,03)$ & $23,00(0,10)$ & $5,90(0,15)$ & $10,333(0,001)$ & 4,6 & $4,50(0,10)$ & $1,0054(0,0001)$ \\
\hline $\mathrm{H} 2$ & Pilsen & ADI & $4,78(0,01)$ & $24,00(0,03)$ & $6,00(0,10)$ & $9,633(0,001)$ & 4,7 & $3,80(0,10)$ & $1,0087(0,0001)$ \\
\hline $\mathrm{I} 2$ & Pilsen & ADI & $4,52(0,01)$ & $24,00(0,02)$ & $6,88(0,10)$ & $10,133(0,001)$ & 4,8 & $4,00(0,10)$ & $1,0057(0,0001)$ \\
\hline $\mathrm{J} 7$ & $\mathrm{~N} / \mathrm{I}$ & $\mathrm{ADI}$ & $4,81(0,01)$ & $18,00(0,05)$ & $6,58(0,10)$ & $9,200(0,001)$ & 4,7 & $4,70(0,05)$ & $1,0075(0,0001)$ \\
\hline K6 & Lager & PMI & $4,75(0,01)$ & $40,00(0,02)$ & $7,95(0,10)$ & $8,100(0,001)$ & 4,4 & $3,90(0,10)$ & $1,0082(0,0001)$ \\
\hline L5 & Pilsen & ADI & $4,82(0,02)$ & $34,00(0,02)$ & $8,33(0,10)$ & $9,316(0,001)$ & 0,0 & $0,00(0,00)$ & $1,0252(0,0001)$ \\
\hline M6 & Pilsen & ADI & $4,60(0,01)$ & $29,00(0,01)$ & $6,30(0,03)$ & $7,750(0,001)$ & 4,7 & $4,60(0,10)$ & $1,0044(0,0001)$ \\
\hline N5 & Pilsen & ADI & $4,70(0,01)$ & $27,00(0,02)$ & $6,45(0,10)$ & $10,950(0,001)$ & 4,5 & $4,50(0,10)$ & $1,0069(0,0001)$ \\
\hline O7 & Pilsen & PMI & $4,91(0,01)$ & $45,00(0,02)$ & $10,35(0,10)$ & $13,700(0,001)$ & 4,7 & $4,50(0,10)$ & $1,0080(0,0001)$ \\
\hline P8 & Pilsen & ADI & $4,76(0,01)$ & $21,00(0,02)$ & $5,40(0,03)$ & $13,500(0,001)$ & 0,0 & $0,00(0,00)$ & $1,0195(0,0001)$ \\
\hline Q9 & $\mathrm{N} / \mathrm{I}$ & PMI & $5,00(0,05)$ & $30,00(0,15)$ & $8,73(0,10)$ & $19,816(0,001)$ & 5,0 & $4,30(0,10)$ & $1,0034(0,0001)$ \\
\hline R2 & Lager & PMI & $4,95(0,01)$ & $48,00(0,02)$ & $10,15(0,05)$ & $14,016(0,001)$ & 5,5 & $4,40(0,10)$ & $1,0082(0,0001)$ \\
\hline $\mathrm{S} 10^{3}$ & Pilsen & PMI & $4,96(0,01)$ & $36,00(0,10)$ & $8,95(0,10)$ & $10,516(0,001)$ & 4,8 & $3,90(0,10)$ & $1,0058(0,0001)$ \\
\hline $\mathrm{T} 8$ & Lager & PMI & $5,00(0,05)$ & $28,00(0,02)$ & $6,08(0,10)$ & $13,350(0,001)$ & 4,6 & $4,40(0,10)$ & $1,0069(0,0001)$ \\
\hline U7 & Lager & PMI & $4,96(0,01)$ & $31,00(0,02)$ & $10,32(0,12)$ & $16,183(0,001)$ & 4,7 & $4,00(0,10)$ & $1,0037(0,0001)$ \\
\hline PPM & Lager & PMA & $4,88(0,01)$ & $32,00(0,02)$ & $11,95(0,25)$ & $12,316(0,001)$ & 4,8 & $5,00(0,05)$ & $1,0077(0,0001)$ \\
\hline $\mathrm{P} 15 \mathrm{M}$ & Lager & $\mathrm{ADA}$ & $4,78(0,02)$ & $33,00(0,02)$ & $10,50(0,10)$ & $11,483(0,001)$ & 4,8 & $4,90(0,02)$ & $1,0097(0,0001)$ \\
\hline P30M & Lager & $\mathrm{ADA}$ & $4,67(0,01)$ & $31,00(0,02)$ & $10,38(0,30)$ & $12,983(0,001)$ & 4,8 & $4,80(0,30)$ & $1,0111(0,0001)$ \\
\hline $\mathrm{P} 45 \mathrm{M}$ & Lager & $\mathrm{ADA}$ & $4,59(0,01)$ & $34,00(0,02)$ & $9,78(0,10)$ & $13,250(0,001)$ & 4,8 & $4,60(0,02)$ & $1,0134(0,0001)$ \\
\hline P15A & Lager & $\mathrm{ADA}$ & $4,80(0,04)$ & $37,00(0,60)$ & $10,30(0,10)$ & $11,900(0,001)$ & 4,8 & $4,90(0,02)$ & $1,0082(0,0001)$ \\
\hline $\mathrm{P} 30 \mathrm{~A}$ & Lager & $\mathrm{ADA}$ & $4,69(0,01)$ & $30,00(0,01)$ & $9,00(0,20)$ & $11,233(0,001)$ & 4,8 & $4,10(0,05)$ & $1,0084(0,0001)$ \\
\hline
\end{tabular}

${ }^{1}$ Puro Malte Industrial (PMI), Adjunto Industrial (ADI), Puro Malte Artesanal (PMA), Adjunto Artesanal (ADA). ${ }^{2}$ Teor alcoólico informado pelo fabricante das cervejas industriais ou estimando para as cervejas artesanais. ${ }^{3}$ Apenas o fabricante da cerveja $\mathrm{S} 10$ informou a intensidade de cor da bebida e amargor da bebida, sendo, respectivamente, de 10 EBC e 11 IBU. 
A determinação da cor permite classificar todas as cervejas estudadas como "claras" (EBC $\leq 20)$. Pode-se observar que, em geral, as cervejas puro malte são aquelas que possuem maior intensidade de cor. Nas amostras artesanais, foi observado que o aumento na concentração de adjuntos pode provocar redução na cor (EBC) da bebida que pode ser associada a menor concentração, no mosto, de aminoácidos e carboidratos derivados do malte os quais sofrem reações de Maillard, conforme descrito anteriormente.

As amostras analisadas possuem amargor que variam entre 7,750 a 19,817 IBU. Observou-se que os valores obtidos para as amostras artesanais indicaram que a adição de milho colaborou para o aumento do amargor da cerveja, enquanto o emprego do arroz como adjunto teve efeito inverso.

Destaca-se que, dentre as amostras industriais, apenas S10 traz informações sobre cor e amargor. Comparando a cor e o amargor experimental/informado pelo fabricante (Tabela 2), observouse que os valores foram próximos, sendo eles, respectivamente, $(8,95 \pm 0,10 / 10,00)$ EBC e $(10,516 \pm 0,001 / 11,000)$ IBU.

Quanto ao teor alcoólico, a legislação brasileira ${ }^{52}$ determina que "cerveja sem álcool" é aquela que possui concentração de álcool $\leq 0,5 \% \mathrm{v} / \mathrm{v}$, não havendo necessidade de constar no rótulo do produto essa informação. Enquanto a "cerveja com álcool" é aquela que possui concentração superior a $0,5 \% \mathrm{v} / \mathrm{v}$, havendo obrigatoriedade em apresentar esse dado na embalagem. Nesse sentido, as amostras analisadas estão situadas na faixa de 0,00 (L5 e P8) a 5,00 \% v/v (PPM e PPM SC), sendo 02 classificadas como cervejas sem álcool (L5 e P8), enquanto as demais são cervejas com álcool.

Uma comparação entre o teor alcoólico experimental/ informado pelo fabricante (Tabela 2) mostra que apenas as cervejas industriais G6 $(4,50 \pm 0,10 / 4,6) \% \mathrm{v} / \mathrm{v}, \mathrm{J} 7(4,70 \pm 0,05 / 4,70) \% \mathrm{v} / \mathrm{v}$, L5 $(0,00 \pm 0,00 / 0,00) \% \mathrm{v} / \mathrm{v}, \mathrm{N} 5(4,50 \pm 0,10 / 4,5) \% \mathrm{v} / \mathrm{v}$ e $\mathrm{P} 8(0,00 \pm 0,00 / 0,00) \% \mathrm{v} / \mathrm{v}$ apresentaram igualdade, dentro do desvio padrão, entre esses valores. Maiores diferenças foram observadas para as bebidas E4 $(4,10 \pm 0,05 / 5,00) \% \mathrm{v} / \mathrm{v}, \mathrm{H} 2(3,80 \pm 0,10 / 4,70) \% \mathrm{v} / \mathrm{v}$, I $2(4,00 \pm 0,10 / 4,80) \% \mathrm{v} / \mathrm{v}, \mathrm{R} 2(4,40 \pm 0,10 / 5,50) \% \mathrm{v} / \mathrm{v}$ e S10 $(3,90 \pm 0,10 / 4,80) \% \mathrm{v} / \mathrm{v}$.

O preparo das amostras artesanais, objetivou a obtenção de bebidas com teor alcoólico igual a 4,80\%v/v (Tabela 1). Os resultados (Tabela 2) indicam maior eficiência na fermentação quando utilizado exclusivamente malte de cevada na mostura, PPM e PPM SC, resultando em bebidas com maior teor alcoólico, respectivamente, $(5,00 \pm 0,05) \% \mathrm{v} / \mathrm{v}$ e $(5,00 \pm 0,08) \% \mathrm{v} / \mathrm{v}$.

Os resultados apontam para uma redução no rendimento alcoólico em função do aumento na concentração $(\% \mathrm{~m} / \mathrm{m})$ de adjuntos (milho e arroz) durante a mostura (Tabela 2). Nas cervejas P15M, P30M e $\mathrm{P} 45 \mathrm{M}$, produzidas com milho, quando comparadas a cerveja puro malte (PPM), observa-se redução no teor alcoólico das bebidas obtendo valores de, respectivamente, $(4,90 \pm 0,02),(4,80 \pm 0,30) \mathrm{e}$ $(4,60 \pm 0,02) \% v / v$. Nota-se ainda uma redução mais acentuada na concentração alcoólica quando utilizado o arroz como adjunto, visto que as amostras P15A, P30A e P45A, apresentaram teores alcoólicos com valores iguais a $(4,90 \pm 0,02),(4,10 \pm 0,05)$ e $(3,40 \pm 0,10) \% \mathrm{v} / \mathrm{v}$, respectivamente. $\mathrm{O}$ malte de cevada possui enzimas $(\alpha$-amilase $\mathrm{e}$ $\beta$-amilase) que atuam na conversão do amido presente no cereal em açúcares fermentáveis e solúveis em água, os quais são convertidos pelas leveduras durante a fermentação em, basicamente, gás carbônico e etanol. A menor concentração de malte pode resultar numa redução de enzimas no mosto e, por consequência, na quantidade de açúcares fermentáveis disponíveis para as leveduras. ${ }^{8}$ Além disso, considerando uma porção de $100 \mathrm{~g}$ de cada cereal, o milho possui maior quantidade de carboidratos $(86,3 \mathrm{~g})$ frente ao $\operatorname{arroz}(79,7 \mathrm{~g}),{ }^{53}$ fator que, combinado as diferentes estruturas dos polissacarídeos que constituem cada cereal, também pode ter influenciado na menor formação de álcool nas cervejas produzidas com esse adjunto (P15A, P30A e P45A).

Adicionalmente, duas amostras de cervejas artesanais foram carbonatadas com sacarose na concentração de $10 \mathrm{~g} \mathrm{~L}^{-1}$, sendo uma puro malte (PPM SC) e uma com $15 \% \mathrm{~m} / \mathrm{m}$ de milho como adjunto (P15M SC). A utilização de sacarose na carbonatação dessas bebidas, em substituição ao extrato de malte, foi realizada com intuito de verificar possíveis alterações na propriedades físicoquímica da bebida, visto que a carbonatação com sacarose é uma técnica comumente utilizada por cervejeiros artesanais devido ao fácil acesso a sacarose em supermercados (açúcar cristal) e ao menor preço quando comparado a outras técnicas.

Foi observado que as cervejas carbonatadas com sacarose, PPM SC e P15M SC, quando comparadas as amostras carbonatadas com extrato de malte, não apresentaram alterações nos valores obtidos para teor alcoólico, mantidos, respectivamente, em 5,00 e 4,90\% \%/v. Contudo, mantendo tal parâmetro de comparação, foi observado aumento no pH e densidade e redução na intensidade de cor, amargor, e acidez total.

As cervejas estudadas, apresentam valor de densidade, a $20^{\circ} \mathrm{C}$, pouco superior à da água, possivelmente devido a presença de açúcares residuais e outros compostos orgânicos dissolvidos na bebida. Nota-se que, dentre as amostras analisadas, as cervejas 0,00\% v/v de álcool (L5 e P8) são as que tem maior valor de densidade, respectivamente, 1,0252 e 1,0195 $\mathrm{g} \mathrm{mL}^{-1}$. O etanol, álcool característico da bebida, possui densidade de $0,7890 \mathrm{~g} \mathrm{~mL}^{-1} \mathrm{a} 20^{\circ} \mathrm{C}$, inferior à da água a mesma temperatura $\left(0,9982 \mathrm{~g} \mathrm{~mL}^{-1}\right)$. Desta forma, a ausência do etanol pode ter colaborado para o aumento na densidade da bebida.

\section{Espectroscopia na Região do Infravermelho}

As análises dos espectros no infravermelho médio (MIR) foram realizadas a partir dos espectros médios (triplicata) das 30 amostras de cervejas selecionadas e produzidas para o estudo (industriais e artesanais), conforme ilustrado na Figura 5. Nela, foram observadas absorções importantes em 3320 e $1640 \mathrm{~cm}^{-1}$, as quais, devido a composição majoritária das amostras, podem ser atribuídas, respectivamente, a sobreposição de sinais associados a deformação angular da ligação $\mathrm{O}-\mathrm{H}$ e deformação axial das ligações $\mathrm{H}-\mathrm{O}-\mathrm{H}$ da água. ${ }^{21}$

Na região de impressão digital (Figura 5), abaixo de $1500 \mathrm{~cm}^{-1}$, foram observadas vibrações de baixa intensidade que podem ser associadas as ligações $\mathrm{C}-\mathrm{C}$ e $\mathrm{C}-\mathrm{H}$ que podem estar associadas aos carboidratos. Além disso, são observadas bandas em 1050-1040 e $875-865 \mathrm{~cm}^{-1}$, que podem ser atribuídas a ligação $\mathrm{C}-\mathrm{O}$ de álcool primário, característico do etanol. ${ }^{21}$ Nas cervejas industriais zero

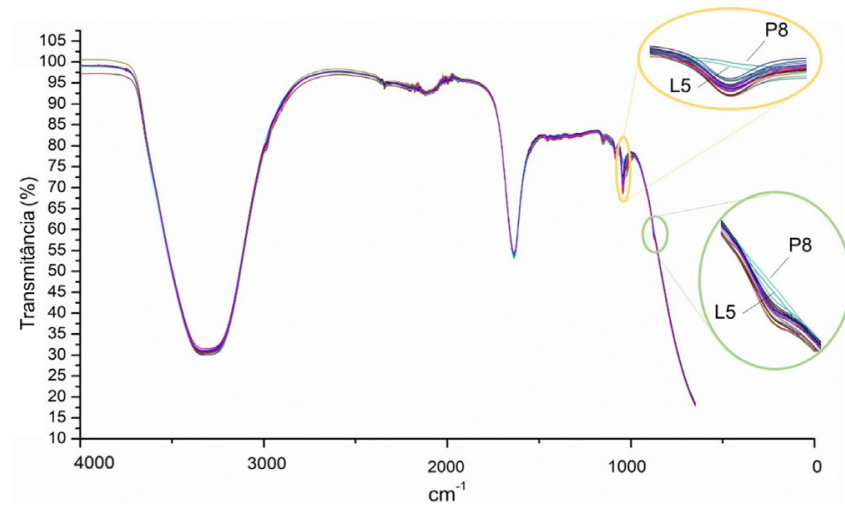

Figura 5. Espectros MIR (4000 a $\left.650 \mathrm{~cm}^{-1}\right)$ das amostras de cervejas analisadas. Destaque para as bandas em 1050-1040 $\mathrm{cm}^{-1}$ (amarelo) e $875-865 \mathrm{~cm}^{-1}$ (verde) 
álcool (L5 e P8), como esperado, não foram observadas essas bandas. Para além disso, na análise visual do espectro MIR, não foi verificada diferenciação composicional entre cervejas industriais e artesanais, nem tampouco entre bebidas puro malte e com adjuntos. Nos espectros (NIR) das trinta amostras de cervejas estudadas, foi possível observar bandas em 6880 e $5115 \mathrm{~cm}^{-1}$, que podem ser associadas a presença de água, componente majoritário da bebida

\section{Espectroscopia de Ressonância Magnética Nuclear}

Para as análises de RMN de ${ }^{1} \mathrm{H}$ foram obtidos espectros com e sem supressão do sinal dos hidrogênios atribuídos a presença de água (4,64 ppm). A supressão do sinal a 4,64 ppm resultou em maior ganho no receptor, possibilitando a melhor identificação de sinais de menor intensidade, em especial, na região dos alifáticos (0 a 5,8 ppm). Por esse motivo, apenas os espectros com supressão em 4,64 ppm foram avaliados no presente trabalho.

Nas amostras de cervejas com álcool (Figura 6.A) verificase, como esperado, a presença dos sinais a 1,03 ppm (tripleto) e 3,5 ppm (quadrupleto) característicos dos hidrogênios metílicos e metilênicos do etanol, respectivamente. Esses sinais apresentam-se com maior intensidade nas amostras, visto que o etanol, após a água, é o componente de maior concentração nessas bebidas. ${ }^{21}$

Além disso, foi observado um dubleto a 1,32 ppm que foi atribuído ao hidrogênio metílico da alanina e, devido a sobreposição de sinais na região de 3 a 4 ppm, não foi possível identificar o sinal referente ao hidrogênio metínico (singleto a 3,77 ppm). Esses compostos também foram observados nos estudos realizados por Almeida e colaboradores, ${ }^{23}$ Rodrigues e colaboradore ${ }^{29}$ e Duarte e colaboradores. ${ }^{21}$

Nas análises realizadas para as cervejas sem álcool (L5 e P8), Figura 6.B, não foram observados sinais relacionados ao etanol (1,03 ppm e 3,50 ppm) e a alanina (1,32 ppm). Porém, foi observado um tripleto a $0,95 \mathrm{ppm}$ e um singleto a $2,20 \mathrm{ppm}$, característicos dos hidrogênios metílicos e da hidroxila do propanol, assim como um dubleto a 1,17 ppm característicos dos hidrogênios metílicos do isopropanol. Esses resultados corroboram com aqueles presentes na literatura científica, ${ }^{29,54,55}$ os quais indicam a existência de propanol e isopropanol (álcoois superiores) em cervejas não alcoólicas.

Cabe destacar que, em nossas análises, devido a sobreposição de sinais nessa região, não foi possível atribuir com precisão sinais que podem ser atribuídos aos álcoois superiores em cervejas com álcool.

Em todo o universo de amostras selecionadas para o presente estudo, foram observados sinais que podem ser atribuídos a ácidos orgânicos, sendo eles produtos da fermentação alcoólica por leveduras, durante a produção de cerveja. A 2,42 ppm foi observado um singleto que foi atribuído aos hidrogênios metilênicos do ácido succínico. Além disso, a 1,32 ppm $(J=6,92 \mathrm{~Hz})$ foi identificado um dubleto associado ao hidrogênio metílico do ácido lático. Por fim, a 1,87 ppm foi observado um singleto atribuído ao hidrogênio metílico do ácido acético. ${ }^{40}$ Ainda foram identificados um dubleto a $7,27 \mathrm{ppm}(J=7,32 \mathrm{~Hz})$ que pode ser associado a um dos sinais da fenilalanina. Atribuídos ao tirosol (derivado do álcool fenetílico) foram identificados dubletos $\mathrm{AB}$ a $6,74 \mathrm{ppm}(J=8,32 \mathrm{~Hz})$ e 7,04 ppm $(J=8,32 \mathrm{~Hz})$, referentes aos hidrogênios aromáticos, e um tripleto a $3,11 \mathrm{ppm}$ referente ao hidrogênio metilênico $(J=6,92 \mathrm{~Hz})$.

A região entre 3-6 ppm é relacionada a sinais de hidrogênios carbinólicos de carboidratos e de alguns álcoois. Contudo, nos espectros obtidos (Figura 6), a presença de sinais sobrepostos nessa região impossibilitou a distinção e atribuição da maioria dos sinais. Dessa forma, foi possível identificar apenas um dubleto a 5,08 ppm $(J=$ $3,64 \mathrm{~Hz}$ ) característico de hidrogênio anomérico, com acoplamento equatorial-axial, indicativo de açúcar com configuração $\alpha$.

\section{ESI $(+) F T-I C R M S$}

A análise dos espectros ESI(+)FT-ICR MS (Figura 7), permitiu a identificação de cátions (Tabela 3 ) que são característicos às amostras analisadas, possibilitando a distinção entre as bebidas industriais e artesanais. Os íons foram atribuídos a moléculas protonadas $\left([\mathrm{M}+\mathrm{H}]^{+}\right)$, adutos de sódio $\left([\mathrm{M}+\mathrm{Na}]^{+}\right)$e de potássio $\left([\mathrm{M}+\mathrm{K}]^{+}\right)$, conforme apresentado na Tabela 3 e ilustrado na Figura 7. Em todos os espectros, foram observados sinais atribuídos aos cátions de maltooligossacarídeos na forma de adutos de sódio e potássio (Tabela 3 e Figura 7). Esses carboidratos derivam da hidrólise do amido presente nos cereais durante mostura dos grãos, indicando que as leveduras não consumiram todos os açucares durante a fermentação e a maturação da bebida. Para todas as amostras foram observados sinais de menor intensidade relacionados à presença de glucose $\left(\mathrm{m} / z 219[\mathrm{M}+\mathrm{K}]^{+}\right)$, maltose/sacarose $\left(\mathrm{m} / z, 365[\mathrm{M}+\mathrm{Na}]^{+}\right.$e $\left.\mathrm{m} / z, 381[\mathrm{M}+\mathrm{K}]^{+}\right)$, maltotriose $\left(\mathrm{m} / z, 543[\mathrm{M}+\mathrm{K}]^{+}\right)$, maltotetraose $\left(\mathrm{m} / z, 705[\mathrm{M}+\mathrm{K}]^{+}\right)$e maltopentaose $\left(\mathrm{m} / z, 867[\mathrm{M}+\mathrm{K}]^{+}\right)$. Os sinais atribuídos a esses açúcares já foram descritos em outros estudos presentes na literatura científica, ${ }^{34}$ corroborando com os resultados apresentados em nossa pesquisa.

Nas amostras artesanais (com exceção de PPM SC e P15M $\mathrm{SC})$ foram identificados sinais de maior intensidade relacionados a compostos derivados da frutose $\left(\mathrm{m} / \mathrm{z}, 673[\mathrm{M}+\mathrm{Na}]^{+}\right)$e galactose/ manose $\left(\mathrm{m} / z, 697[\mathrm{M}+\mathrm{Na}]^{+}\right)$. É importante destacar que nessas
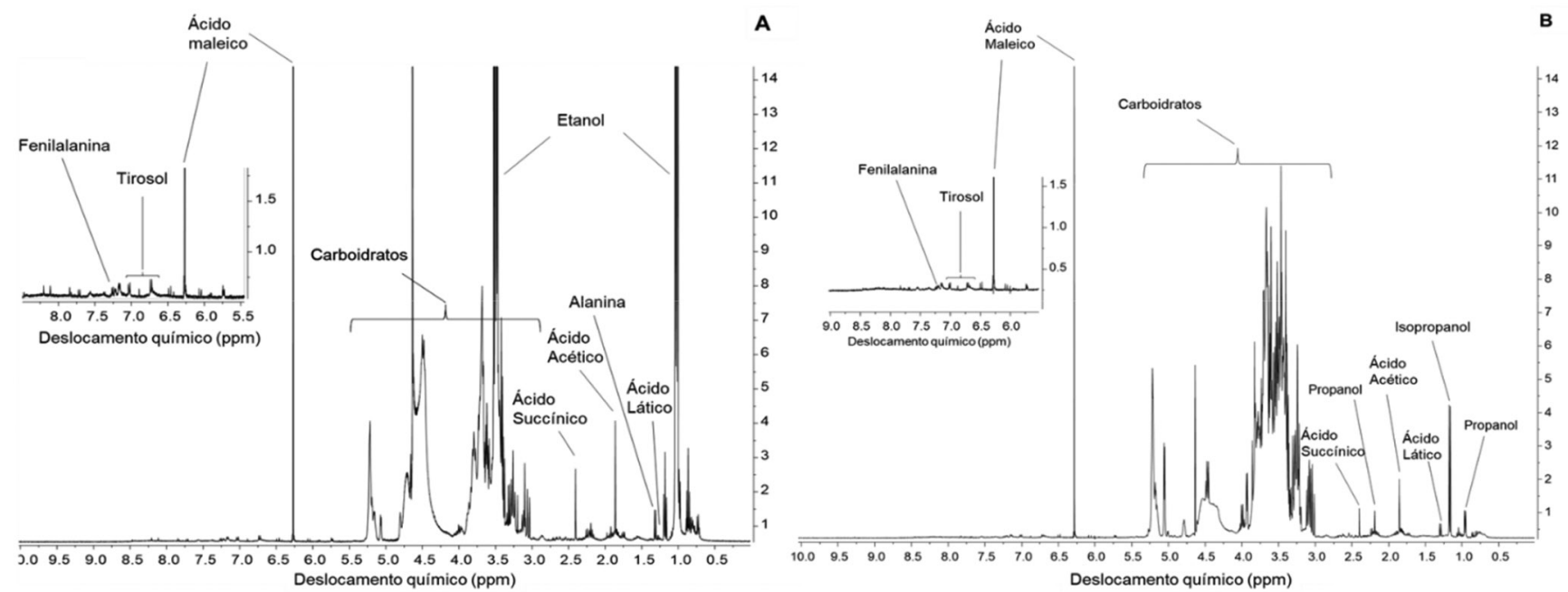

Figura 6. Espectros de RMN de ${ }^{1} \mathrm{H}$ com destaque aos compostos característicos presentes nas amostras de cervejas estudadas. (A) Cerveja com álcool-Amostra A1. (B) Cerveja sem álcool-Amostra L5 
amostras também foram identificados sinais atribuídos ao inostamycin B $\left(\mathrm{m} / z, 709[\mathrm{M}+\mathrm{Na}]^{+}\right)$, isolado originalmente da bactéria Streptomyces $s p$. MH816- AF1542, ${ }^{56,57}$ e ao TMC-151C $\left(\mathrm{m} / \mathrm{z}, 797[\mathrm{M}+\mathrm{Na}]^{+}\right)$, isolado inicialmente do fungo Gliocladium catenulatum ${ }^{58,59}$ ambos os compostos com atividade antibiótica. Em nossa busca na literatura científica, esse é o primeiro relato da presença destes compostos em cervejas e, possivelmente, reflete a contribuição do método de cultivo dos grãos utilizados na produção da bebida (malte de cevada e/ou extrato de malte, visto que a Streptomyces $s p$ e a Gliocladium catenulatum podem ser empregadas no controle de patógenos característicos em cultivares de milho, cevada, trigo, etc..$^{60,61}$

Além de açúcares, durante a etapa de fermentação, as leveduras demandam em seu metabolismo compostos nitrogenados, sendo suas principais fontes os aminoácidos, proteínas e peptídeos. ${ }^{62}$ Os compostos nitrogenados estão presentes em quantidades satisfatórias no malte de cevada, os quais são solubilizados no mosto durante a hidrólise dos carboidratos. ${ }^{63} \mathrm{~A}$ presença de amino álcoois, purinas e pirimidinas em cervejas é esperada, visto que são metabólitos das leveduras, e, ao final da fermentação, estima-se que sejam exauridos a maioria dos aminoácidos e proteínas, restando apenas a prolina. ${ }^{54}$

Os sinais identificados, apresentados na Tabela 3 e Figura 7 , mostram que os sinais $[\mathrm{M}+\mathrm{H}]^{+}$de maior intensidade podem estar associados, a amino álcoois $(\mathrm{m} / \mathrm{z}, 218,230,246,262,362,386$ e 406 $\left.[\mathrm{M}+\mathrm{H}]^{+}\right)$, purinas $\left(\mathrm{m} / z 268,369\right.$ e $\left.401[\mathrm{M}+\mathrm{H}]^{+}\right)$, pirimidinas $(\mathrm{m} / \mathrm{z} 317$ e $\left.785[\mathrm{M}+\mathrm{H}]^{+}\right)$, esfingolipídios $\left(\mathrm{m} / z, 274\right.$ e $\left.318[\mathrm{M}+\mathrm{H}]^{+}\right)$piperazinas $\left(\mathrm{m} / \mathrm{z} 397\right.$ e $\left.445[\mathrm{M}+\mathrm{H}]^{+}\right)$, proteínas $(\mathrm{m} / \mathrm{z}, 477,497,521,533,609$, $629,653,853$ e $\left.1077[\mathrm{M}+\mathrm{H}]^{+}\right)$e aminoácidos $(\mathrm{m} / \mathrm{z}, 391,440,532$, $541,570,635,663,756$ e $\left.798[\mathrm{M}+\mathrm{H}]^{+}\right) .{ }^{64}$ Neste contexto, destaca-se a maior quantidade e intensidade de sinais atribuídos a aminoácidos e proteínas nas cervejas artesanais (Figura 7.B), com exceção de PPM SC e P15M SC, as quais possuem perfil semelhante as cervejas industriais (Figura 7.A). Considerando que todas as cervejas artesanais seguiram as mesmas condições durante sua produção, uma possível explicação para a semelhança entre o perfil químico das amostras PPM SC e P15M SC com o das amostras industriais pode estar relacionada à sua carbonatação. Pois, ambas amostras foram carbonatadas por refermentação com adição de sacarose. Nesse processo, as leveduras consomem o açúcar adicionado, gaseificando a bebida, e junto a ele os compostos nitrogenados ainda presentes na bebida. Enquanto as demais amostras artesanais foram carbonatadas por refermentação com adição de extrato de malte, proporcionando a essas leveduras quantidade extra de açucares, mas também de compostos nitrogenados naturalmente presentes no malte, dentre eles proteínas e aminoácidos, aumentando sua concentração na bebida, quando comparada as carbonatadas com sacarose.
Em geral, os processos produtivos industriais buscam maior eficiência no menor tempo possível. Possivelmente, as bebidas das cervejarias industrias utilizam meios produtivos que visam acelerar o processo de fermentação buscando alta eficiência na conversão dos açúcares presentes no mosto, resultando também em bebidas com menor presença de aminoácidos e proteínas. É importante destacar que a maior presença de proteínas e aminoácidos resulta em bebidas com melhor qualidade nutricional. ${ }^{65}$

Em adicional, foi observado no espectro das amostras A1, B2 e U7, sinal que foi atribuído ao Piriminobac $\left(\mathrm{m} / \mathrm{z} 348[\mathrm{M}+\mathrm{H}]^{+}\right)$, um éter aromático que possui ação herbicida e que pode ser utilizado no controle de vegetações indesejáveis. Sua aplicação, assim como sua estrutura metilada (Piriminobac-metil), é relatada em plantações de cereais para consumo humano, tais como a cevada, milho, arroz, cana-de-açúcar, etc. A presença do Piriminobac em cervejas aponta para a persistência desse herbicida, mesmo quando submetido ao ciclo industrial da bebida. ${ }^{66}$ Ressaltamos que, em nossas análises, foi avaliado apenas um lote de cada bebida, indicando a necessidade de avaliações posteriores acerca da presença desse composto em outros lotes dos rótulos citados, visto que esse pode ser um resultado pontual.

\section{ESI(-)FT-ICR MS}

A Figura 8 ilustra os espectros típicos obtidos para as cervejas industriais (Figura 8.A) e as artesanais (Figura 8.B), tendo o resumo dos principais íons detectados apresentados na Tabela 4. Nos espectros das 30 amostras foram observados sinais atribuídos aos ânions presentes na bebida, os quais foram associados as moléculas em suas formas desprotonadas ([M-H $\left.]^{-}\right)$e também a adutos de cloreto ([M-Cl $]^{-}$) no padrão isotópico típico ${ }^{35} \mathrm{Cl} \mathrm{e}{ }^{37} \mathrm{Cl} .{ }^{33}$

Os resultados obtidos pelo emprego de ESI(-) corroboram com aqueles apresentados no ESI(+). Em todas as amostras foram observados sinais associados a presença de açucares, sendo os ânions $\mathrm{m} / \mathrm{z} 161,179$ e 191 atribuídos, respectivamente, as formas [M-H] ${ }^{-} \mathrm{da}$ 1,6 -anidro- $\beta$-D-glicose, glicose e D-glucaro-1,4-lactona. Enquanto os pares de ânions $m / z$ 215/217, 377/379 e 549/541 foram relacionados, respectivamente, a $[\mathrm{M}-\mathrm{Cl}]^{-}$da glicose, maltose e maltotriose.

O emprego do ESI (-) possibilitou a identificação de compostos característicos da cerveja, complementando os resultados do ESI(+). Em todas as bebidas foram identificados os ânions $\mathrm{m} / \mathrm{z} 347$ e 361, atribuídos as formas desprotonadas da humulona e seus isômeros. Esses compostos derivam do lúpulo e são responsáveis pelo amargor da cerveja, além de contribuir no aroma, estabilização e conservação da bebida.

A análise dos dados apresentados na Figura 8 e na Tabela 4 apontam para uma predominância de sinais relacionados a açúcares nas bebidas comerciais, enquanto nas cervejas artesanais analisadas
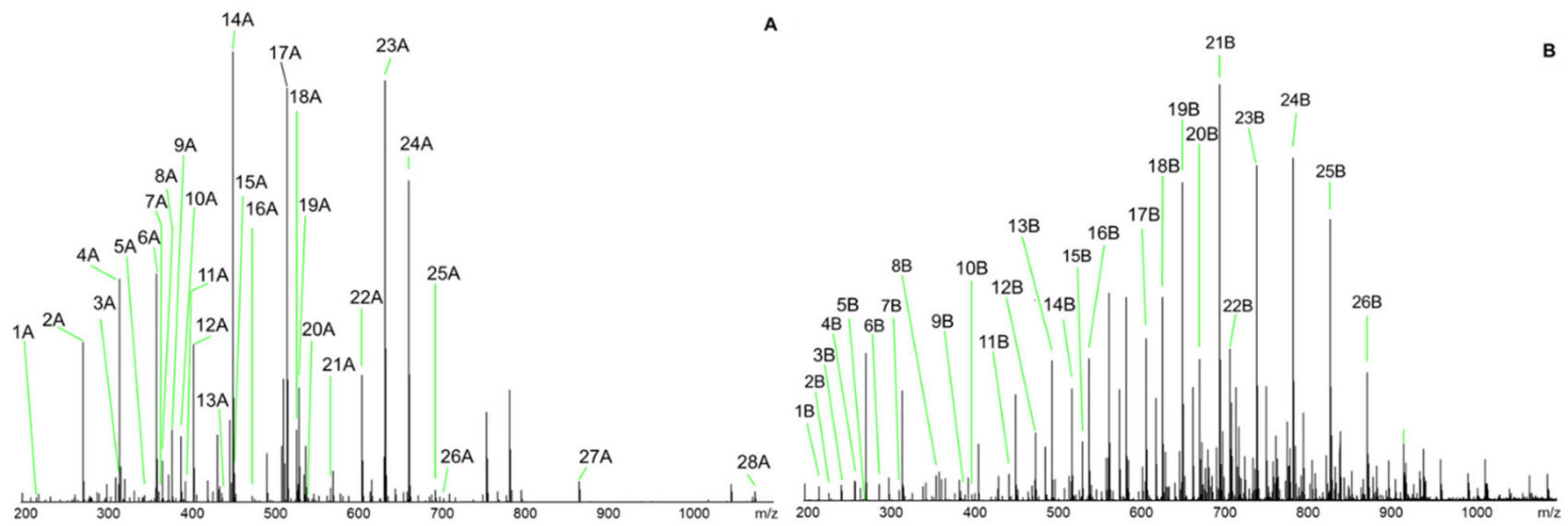

Figura 7. Espectros de impressão digital IES(+) representativos das amostras de cervejas industriais (A) e cervejas artesanais(B). Sinais identificados na Tabela 3 
Tabela 3. Resumo dos principais íons detectados no IES(+). Cervejas Puro Malte Industrial (PMI), Adjunto Industrial (ADI), Puro Malte Artesanal (PMA), Adjunto Artesanal (ADA). *Inclui PPM SC e P15M SC. 'Exceto PPM SC e P15M SC

\begin{tabular}{|c|c|c|c|c|c|c|c|}
\hline $\mathrm{N}^{\circ}$ & $m / z$ & Forma do sinal & Estrutura proposta & PMI* & ADI & PMA & $\mathrm{ADA}^{\gamma}$ \\
\hline$\overline{1 B}$ & 218,21135 & {$\left[\mathrm{C}_{12} \mathrm{H}_{27} \mathrm{NO}_{2}+\mathrm{H}\right]^{+}$} & 2-[2-hidroxietil(octil)amino]etanol & & & $\mathrm{X}$ & $\mathrm{X}$ \\
\hline $1 \mathrm{~A}$ & 219,03773 & {$\left[\mathrm{C}_{6} \mathrm{H}_{12} \mathrm{O}_{6}+\mathrm{K}\right]^{+}$} & Glicose & $\mathrm{X}$ & $\mathrm{X}$ & & \\
\hline $2 \mathrm{~B}$ & 230,24777 & {$\left[\mathrm{C}_{14} \mathrm{H}_{31} \mathrm{NO}+\mathrm{H}\right]^{+}$} & 2-(Dodecilamino)etanol & & & $\mathrm{X}$ & \\
\hline $3 \mathrm{~B}$ & 246,24268 & {$\left[\mathrm{C}_{14} \mathrm{H}_{31} \mathrm{NO}_{2}+\mathrm{H}\right]^{+}$} & 1-[2-hidroxipropil(octil)amino]propan-2-ol & & & $\mathrm{X}$ & $\mathrm{X}$ \\
\hline 4B & 262,23761 & {$\left[\mathrm{C}_{14} \mathrm{H}_{31} \mathrm{NO}_{3}+\mathrm{H}\right]^{+}$} & 2-[Bis(3-metilbutil)amino]-2-(hidroxiImetil)propan)-1,3-diol & & & $\mathrm{X}$ & $\mathrm{X}$ \\
\hline $5 \mathrm{~B}$ & 268,10399 & {$\left[\mathrm{C}_{10} \mathrm{H}_{13} \mathrm{~N}_{5} \mathrm{O}_{4}+\mathrm{H}\right]^{+}$} & Adenosina ou Vidarabina & & & $\mathrm{X}$ & \\
\hline $2 \mathrm{~A}$ & 274,27399 & {$\left[\mathrm{C}_{16} \mathrm{H}_{35} \mathrm{NO}_{2}+\mathrm{H}\right]^{+}$} & Hexadecasfinganina & $\mathrm{X}$ & $\mathrm{X}$ & $\mathrm{X}$ & $\mathrm{X}$ \\
\hline $6 \mathrm{~B}$ & 290,26891 & {$\left[\mathrm{C}_{16} \mathrm{H}_{35} \mathrm{~N}_{3}+\mathrm{H}\right]^{+}$} & 3-((2-hidroxiIdodecil)metilamina)propano-1,2-diol & $\mathrm{X}$ & $\mathrm{X}$ & $\mathrm{X}$ & $\mathrm{X}$ \\
\hline $7 \mathrm{~B}$ & 314,24172 & {$\left[\mathrm{C}_{20} \mathrm{H}_{44} \mathrm{NO}+\mathrm{H}\right]^{+}$} & N-decildecan-1-amina & $\mathrm{X}$ & $\mathrm{X}$ & $\mathrm{X}$ & $\mathrm{X}$ \\
\hline $3 \mathrm{~A}$ & 317,12175 & {$\left[\mathrm{C}_{17} \mathrm{H}_{12} \mathrm{~N}_{6} \mathrm{O}+\mathrm{H}\right]^{+}$} & Pirimidinas & $\mathrm{X}$ & $\mathrm{X}$ & & $\mathrm{X}$ \\
\hline $4 \mathrm{~A}$ & 318,30021 & {$\left[\mathrm{C}_{18} \mathrm{H}_{39} \mathrm{NO}_{3}+\mathrm{H}\right]^{+}$} & Fitosfingosina & $\mathrm{X}$ & $\mathrm{X}$ & $\mathrm{X}$ & $X$ \\
\hline $5 \mathrm{~A}$ & 348,12347 & {$\left[\mathrm{C}_{16} \mathrm{H}_{17} \mathrm{~N}_{3} \mathrm{O}_{6}+\mathrm{H}\right]^{+}$} & Piriminobac & & & & \\
\hline $8 \mathrm{~B}$ & 358,36790 & {$\left[\mathrm{C}_{22} \mathrm{H}_{47} \mathrm{NO}_{2}+\mathrm{H}\right]^{+}$} & Estearildietanolamina & & & $\mathrm{X}$ & $X$ \\
\hline $6 \mathrm{~A}$ & 362,33641 & {$\left[\mathrm{C}_{20} \mathrm{H}_{43} \mathrm{NO}_{4}+\mathrm{H}\right]^{+}$} & Amino álcoois & $\mathrm{X}$ & $\mathrm{X}$ & $\mathrm{X}$ & $\mathrm{X}$ \\
\hline $7 \mathrm{~A}$ & 365,11717 & {$\left[\mathrm{C}_{12} \mathrm{H}_{22} \mathrm{O}_{11}+\mathrm{Na}\right]^{+}$} & Maltose/sacarose & $\mathrm{X}$ & $\mathrm{X}$ & $\mathrm{X}$ & $X$ \\
\hline $8 \mathrm{~A}$ & 369,20011 & {$\left[\mathrm{C}_{19} \mathrm{H}_{24} \mathrm{~N}_{6} \mathrm{O}_{2}+\mathrm{H}\right]^{+}$} & Purinas & $\mathrm{X}$ & $\mathrm{X}$ & $\mathrm{X}$ & $X$ \\
\hline $9 \mathrm{~A}$ & 381,08744 & {$\left[\mathrm{C}_{12} \mathrm{H}_{22} \mathrm{O}_{11}+\mathrm{K}\right]^{+}$} & Maltose/sacarose & $\mathrm{X}$ & $\mathrm{X}$ & $\mathrm{X}$ & $\mathrm{X}$ \\
\hline 9B & 386,39921 & {$\left[\mathrm{C}_{24} \mathrm{H}_{51} \mathrm{NO}_{2}+\mathrm{H}\right]^{+}$} & 1-[2-hidroxipropil(octadecil)amino]propan-2-ol & & & $\mathrm{X}$ & $\mathrm{X}$ \\
\hline $10 \mathrm{~A}$ & 391,35458 & {$\left[\mathrm{C}_{21} \mathrm{H}_{46} \mathrm{~N}_{2} \mathrm{O}_{4}+\mathrm{H}\right]^{+}$} & 凶-alanina & $\mathrm{X}$ & $\mathrm{X}$ & $\mathrm{X}$ & $\mathrm{X}$ \\
\hline $11 \mathrm{~A}$ & 397,27145 & {$\left[\mathrm{C}_{22} \mathrm{H}_{32} \mathrm{~N}_{6} \mathrm{O}+\mathrm{H}\right]^{+}$} & Piperazinas & $\mathrm{X}$ & $\mathrm{X}$ & $\mathrm{X}$ & $\mathrm{X}$ \\
\hline 10B & 401,26640 & {$\left[\mathrm{C}_{21} \mathrm{H}_{32} \mathrm{~N}_{6} \mathrm{O}_{2}+\mathrm{H}\right]^{+}$} & Purinas & & & $\mathrm{X}$ & $\mathrm{X}$ \\
\hline $12 \mathrm{~A}$ & 406,35785 & {$\left[\mathrm{C}_{22} \mathrm{H}_{47} \mathrm{NO}_{5}+\mathrm{H}\right]^{+}$} & Aminopentol & $\mathrm{X}$ & $\mathrm{X}$ & & \\
\hline $13 \mathrm{~A}$ & 440,25896 & {$\left[\mathrm{C}_{20} \mathrm{H}_{34} \mathrm{~N}_{5} \mathrm{O}_{6}+\mathrm{H}\right]^{+}$} & Aminoácidos & $\mathrm{X}$ & $\mathrm{X}$ & $\mathrm{X}$ & $\mathrm{X}$ \\
\hline 11B & 445,29257 & {$\left[\mathrm{C}_{23} \mathrm{H}_{36} \mathrm{~N}_{6} \mathrm{O}_{3}+\mathrm{H}\right]^{+}$} & Piperazinas & & & $\mathrm{X}$ & $\mathrm{X}$ \\
\hline $14 \mathrm{~A}$ & 453,13964 & {$\left[\mathrm{C}_{25} \mathrm{H}_{20} \mathrm{~N}_{6} \mathrm{O}_{3}+\mathrm{H}\right]^{+}$} & Quinoxalinas & $\mathrm{X}$ & $\mathrm{X}$ & $\mathrm{X}$ & $\mathrm{X}$ \\
\hline $15 \mathrm{~A}$ & 455,17765 & {$\left[\mathrm{C}_{20} \mathrm{H}_{20} \mathrm{~N}_{2} \mathrm{O}_{10}+\mathrm{H}\right]^{+}$} & Diaminofenil Glicosilado & $\mathrm{X}$ & $\mathrm{X}$ & $\mathrm{X}$ & $\mathrm{X}$ \\
\hline $16 \mathrm{~A}$ & 476,26650 & {$\left[\mathrm{C}_{26} \mathrm{H}_{37} \mathrm{NO}_{7}+\mathrm{H}\right]^{+}$} & Derivado do Orto-catecol & $\mathrm{X}$ & $\mathrm{X}$ & $\mathrm{X}$ & $\mathrm{X}$ \\
\hline $12 \mathrm{~B}$ & 477,28243 & {$\left[\mathrm{C}_{23} \mathrm{H}_{36} \mathrm{~N}_{6} \mathrm{O}_{5}+\mathrm{H}\right]^{+}$} & Proteína derivada da L-alanina, L-tirosina, L-leucina ou norvalina & & & $\mathrm{X}$ & $\mathrm{X}$ \\
\hline $13 \mathrm{~B}$ & 497,23590 & {$\left[\mathrm{C}_{21} \mathrm{H}_{32} \mathrm{~N}_{6} \mathrm{O}_{8}+\mathrm{H}\right]^{+}$} & Proteína derivada da L-histidina ou L-prolina & & & $\mathrm{X}$ & $\mathrm{X}$ \\
\hline $17 \mathrm{~A}$ & 518,31147 & {$\left[\mathrm{C}_{29} \mathrm{H}_{43} \mathrm{NO}_{7}+\mathrm{H}\right]^{+}$} & Estrofantina-3-dimetilamino acetato & $\mathrm{X}$ & $\mathrm{X}$ & & \\
\hline 14B & 521,30867 & {$\left[\mathrm{C}_{25} \mathrm{H}_{40} \mathrm{~N}_{6} \mathrm{O}_{6}+\mathrm{H}\right]^{+}$} & Proteína derivada da L-alanina ou L-leucina. & & & $\mathrm{X}$ & $\mathrm{X}$ \\
\hline $18 \mathrm{~A}$ & 530,27489 & {$\left[\mathrm{C}_{29} \mathrm{H}_{39} \mathrm{NO}_{8}+\mathrm{H}\right]^{+}$} & Ésteres & $\mathrm{X}$ & $\mathrm{X}$ & $\mathrm{X}$ & $\mathrm{X}$ \\
\hline $19 \mathrm{~A}$ & 532,29502 & {$\left[\mathrm{C}_{29} \mathrm{H}_{41} \mathrm{NO}_{8}+\mathrm{H}\right]^{+}$} & Aminoácidos & $\mathrm{X}$ & $\mathrm{X}$ & $\mathrm{X}$ & $\mathrm{X}$ \\
\hline 15B & 533,34502 & {$\left[\mathrm{C}_{27} \mathrm{H}_{44} \mathrm{~N}_{6} \mathrm{O}_{5}+\mathrm{H}\right]^{+}$} & Proteína derivada da L-prolina ou L-leucina. & & & $\mathrm{X}$ & $\mathrm{X}$ \\
\hline $16 \mathrm{~B}$ & 541,26217 & {$\left[\mathrm{C}_{23} \mathrm{H}_{36} \mathrm{~N}_{6} \mathrm{O}_{9}+\mathrm{H}\right]^{+}$} & Proteína derivada da L-fenilalanina & & & $\mathrm{X}$ & $\mathrm{X}$ \\
\hline $20 \mathrm{~A}$ & 543,13020 & {$\left[\mathrm{C}_{18} \mathrm{H}_{32} \mathrm{O}_{16}+\mathrm{K}\right]^{+}$} & Maltotriose & $\mathrm{X}$ & $\mathrm{X}$ & $\mathrm{X}$ & $\mathrm{X}$ \\
\hline $21 \mathrm{~A}$ & 570,25589 & {$\left[\mathrm{C}_{30} \mathrm{H}_{31} \mathrm{~N}_{7} \mathrm{O}_{5}+\mathrm{H}\right]^{+}$} & Derivados da norleucina e glutamina & $\mathrm{X}$ & $\mathrm{X}$ & $\mathrm{X}$ & $\mathrm{X}$ \\
\hline $22 \mathrm{~A}$ & 607,47325 & {$\left[\mathrm{C}_{36} \mathrm{H}_{58} \mathrm{~N}_{6} \mathrm{O}_{2}+\mathrm{H}\right]^{+}$} & Isoquinolinas & $\mathrm{X}$ & $\mathrm{X}$ & $\mathrm{X}$ & $\mathrm{X}$ \\
\hline 17B & 609,36115 & {$\left[\mathrm{C}_{29} \mathrm{H}_{48} \mathrm{~N}_{6} \mathrm{O}_{8}+\mathrm{H}\right]^{+}$} & Proteínas derivadas da L-lisina, L-isoleucina ou L-leucina & & & $\mathrm{X}$ & $X$ \\
\hline 18B & 629,31459 & {$\left[\mathrm{C}_{27} \mathrm{H}_{44} \mathrm{~N}_{6} \mathrm{O}_{11}+\mathrm{H}\right]^{+}$} & L-Alanil-L-leucil-L- $\alpha$-glutamil-L- $\alpha$-glutamil-D-prolil-L-alanina & & & $\mathrm{X}$ & $\mathrm{X}$ \\
\hline $23 \mathrm{~A}$ & 635,50456 & {$\left[\mathrm{C}_{38} \mathrm{H}_{62} \mathrm{~N}_{6} \mathrm{O}_{2}+\mathrm{H}\right]^{+}$} & Aminoácidos & $\mathrm{X}$ & $\mathrm{X}$ & $\mathrm{X}$ & $\mathrm{X}$ \\
\hline 19B & 653,38732 & $\mathrm{C}_{32} \mathrm{H}_{48} \mathrm{~N}_{10} \mathrm{O}_{5}$ & Proteínas derivadas da $\beta$-alanina, L-valina e L-norleucina & & & $\mathrm{X}$ & $\mathrm{X}$ \\
\hline $24 \mathrm{~A}$ & 663,53859 & {$\left[\mathrm{C}_{40} \mathrm{H}_{66} \mathrm{~N}_{6} \mathrm{O}_{2}+\mathrm{H}\right]^{+}$} & Derivado da L-lisina & $\mathrm{X}$ & $\mathrm{X}$ & $\mathrm{X}$ & $\mathrm{X}$ \\
\hline 20B & 673.34077 & {$\left[\mathrm{C}_{31} \mathrm{H}_{54} \mathrm{O}_{14}+\mathrm{Na}\right]^{+}$} & Frutooligossacarídeos & & & $\mathrm{X}$ & $\mathrm{X}$ \\
\hline $25 \mathrm{~A}$ & 695,39777 & {$\left[\mathrm{C}_{40} \mathrm{H}_{50} \mathrm{~N}_{6} \mathrm{O}_{5}+\mathrm{H}\right]^{+}$} & Derivados de Carbamato de benzila & $\mathrm{X}$ & $\mathrm{X}$ & & \\
\hline 21B & 697,41355 & {$\left[\mathrm{C}_{35} \mathrm{H}_{62} \mathrm{O}_{12}+\mathrm{Na}\right]^{+}$} & Oligossacarídeo derivado da galactose/manose & & & $\mathrm{X}$ & $\mathrm{X}$ \\
\hline 22B & 709,45000 & {$\left[\mathrm{C}_{37} \mathrm{H}_{66} \mathrm{O}_{11}+\mathrm{Na}\right]^{+}$} & Inostamycin B & & & $\mathrm{X}$ & $\mathrm{X}$ \\
\hline $26 \mathrm{~A}$ & 705,64548 & {$\left[\mathrm{C}_{24} \mathrm{H}_{42} \mathrm{O}_{21}+\mathrm{K}\right]^{+}$} & Maltotetraose & $\mathrm{X}$ & $\mathrm{X}$ & $\mathrm{X}$ & $\mathrm{X}$ \\
\hline $23 \mathrm{~B}$ & 741,44978 & {$\left[\mathrm{C}_{36} \mathrm{H}_{56} \mathrm{~N}_{10} \mathrm{O}_{7}+\mathrm{H}\right]^{+}$} & Proteínas & & & $\mathrm{X}$ & $\mathrm{X}$ \\
\hline 24B & 785,46599 & {$\left[\mathrm{C}_{37} \mathrm{H}_{65} \mathrm{~N}_{6} \mathrm{O}_{12}+\mathrm{H}\right]^{+}$} & Nucleosídeos de pirimidinas & & & $\mathrm{X}$ & $\mathrm{X}$ \\
\hline $25 \mathrm{~B}$ & 797,50231 & {$\left[\mathrm{C}_{41} \mathrm{H}_{74} \mathrm{O}_{15}+\mathrm{Na}\right]^{+}$} & TMC-151C & & & $\mathrm{X}$ & $\mathrm{X}$ \\
\hline $27 \mathrm{~A}$ & 867,37193 & {$\left[\mathrm{C}_{30} \mathrm{H}_{52} \mathrm{O}_{26}+\mathrm{K}\right]^{+}$} & Maltopentaose & $\mathrm{X}$ & $\mathrm{X}$ & $\mathrm{X}$ & $\mathrm{X}$ \\
\hline $26 \mathrm{~B}$ & 853,21222 & {$\left[\mathrm{C}_{42} \mathrm{H}_{68} \mathrm{~N}_{10} \mathrm{O}_{10}+\mathrm{H}\right]^{+}$} & Proteína (L-Lisil-L-leucil-L-tirosil-L-histidil-L-leucil-L-seril-L-isoleucina) & & & $\mathrm{X}$ & $\mathrm{X}$ \\
\hline $28 \mathrm{~A}$ & 1077,74354 & {$\left[\mathrm{C}_{49} \mathrm{H}_{92} \mathrm{~N}_{18} \mathrm{O}_{9}+\mathrm{H}\right]^{+}$} & Proteínas derivadas da norleucina, leucina, lisina e prolina & $\mathrm{X}$ & $\mathrm{X}$ & $\mathrm{X}$ & $\mathrm{X}$ \\
\hline
\end{tabular}


há a predominância de ésteres, ácidos carboxílicos e outros compostos que contribuem positivamente para o aroma da bebida.

\section{Análise Quimiométrica}

A partir dos dados experimentais obtidos por NIR, MIR, RMN de ${ }^{1} \mathrm{H}$ e ESI( \pm )FT-ICR MS, foram construídos modelos quimiométricos com intuito de correlacionar os espectros das amostras de cerveja com suas propriedades físico-químicas. A melhor fonte de dados, modelo e parâmetros de desempenho e de acurácia são apresentados na Tabela 5
Dentre os resultados apresentados na Tabela 5, o modelo PLS apresentou melhores métricas para modelagem das propriedades físico-químicas de amargor e teor alcoólico, enquanto o modelo SVR obteve melhor métrica para estimativa de densidade das amostras de cerveja. Esses modelos apresentaram percentuais de acerto, acima de $84 \%$ na calibração e de $61 \%$ no grupo de teste, somados com limites de determinação e quantificação. Já quanto às propriedades de $\mathrm{pH}$, acidez total e cor, os modelos PLS construídos apresentaram percentuais de acerto superior a $77 \%$, porém inferiores a $31 \%$ no grupo de teste. Nota-se que a maioria das propriedades apresentaram boa linearidade, com exceção da propriedade de acidez total.
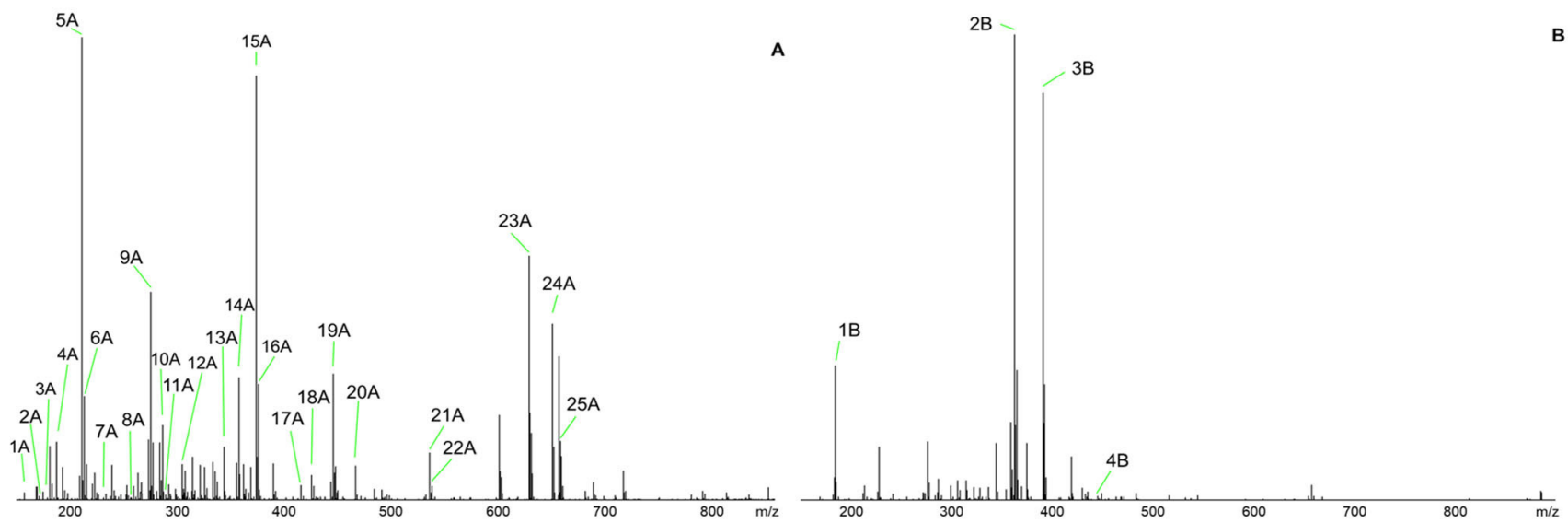

Figura 8. Espectros de impressão digital IES(-) representativos das amostras de cervejas industriais (A) e cervejas artesanais (B). Sinais identificados na Tabela 4

Tabela 4. Resumo dos principais íons detectados no IES(-). Cervejas Puro Malte Industrial (PMI), Adjunto Industrial (ADI), Puro Malte Artesanal (PMA), Adjunto Artesanal (ADA). *Inclui PPM SC e P15M SC. 'Exceto PPM SC e P15M SC

\begin{tabular}{|c|c|c|c|c|c|c|c|}
\hline $\mathrm{N}^{\circ}$ & $\mathrm{m} / \mathrm{z}$ & Forma do sinal & Estrutura proposta & PMI* & ADI & PMA & $\mathrm{ADA}^{\gamma}$ \\
\hline$\overline{1 \mathrm{~A}}$ & 161,04557 & {$\left[\mathrm{C}_{6} \mathrm{H}_{10} \mathrm{O}_{5}-\mathrm{H}\right]^{-}$} & 1,6-Anidro- $\beta$-D-glicose & $\mathrm{X}$ & $\mathrm{X}$ & & \\
\hline $2 \mathrm{~A}$ & 169,01748 & {$\left[\mathrm{C}_{7} \mathrm{H}_{5} \mathrm{O}_{5}-\mathrm{H}\right]^{-}$} & Ácido Gálico & $\mathrm{X}$ & $\mathrm{X}$ & & \\
\hline $3 \mathrm{~A}$ & 179,05618 & {$\left[\mathrm{C}_{6} \mathrm{H}_{12} \mathrm{O}_{6}-\mathrm{H}\right]^{-}$} & Glicose & $\mathrm{X}$ & $\mathrm{X}$ & & \\
\hline 1B & 188,03101 & {$\left[\mathrm{C}_{10} \mathrm{H}_{7} \mathrm{NO}_{3}-\mathrm{H}\right]^{-}$} & Ácido 3-hidroxiquinolina-4-carboxílico ${ }^{186}$ & & & $\mathrm{X}$ & $\mathrm{X}$ \\
\hline $4 \mathrm{~A}$ & 191,01979 & {$\left[\mathrm{C}_{6} \mathrm{H}_{8} \mathrm{O}_{7}-\mathrm{H}\right]^{-}$} & D-Glucaro-1,4-lactona & $\mathrm{X}$ & $\mathrm{X}$ & $\mathrm{X}$ & $\mathrm{X}$ \\
\hline $5 \mathrm{~A}$ & 215,03443 & {$\left[\mathrm{C}_{6} \mathrm{H}_{12} \mathrm{O}_{6+} \mathrm{Cl}\right]^{-}$} & Glicose & $X$ & $\mathrm{X}$ & $\mathrm{X}$ & $X$ \\
\hline $6 \mathrm{~A}$ & 217,03993 & {$\left[\mathrm{C}_{6} \mathrm{H}_{12} \mathrm{O}_{6+} \mathrm{Cl}\right]^{-}$} & Glicose & $\mathrm{X}$ & $\mathrm{X}$ & $\mathrm{X}$ & $\mathrm{X}$ \\
\hline 7A & 233,10485 & {$\left[\mathrm{C}_{10} \mathrm{H}_{17} \mathrm{O}_{6}-\mathrm{H}\right]^{-}$} & Diisopropil D-tartarato & & & $\mathrm{X}$ & $\mathrm{X}$ \\
\hline $8 \mathrm{~A}$ & 271,05432 & {$\left[\mathrm{C}_{11} \mathrm{H}_{8} \mathrm{~N}_{6} \mathrm{O}_{3}-\mathrm{H}\right]^{-}$} & 8-(p-nitrofenil)guanina & & & $\mathrm{X}$ & \\
\hline $9 \mathrm{~A}$ & 279,03792 & {$\left[\mathrm{C}_{9} \mathrm{H}_{12} \mathrm{~N}_{2} \mathrm{O}_{6}+\mathrm{Cl}\right]^{-}$} & Uridina & $\mathrm{X}$ & $\mathrm{X}$ & $\mathrm{X}$ & $\mathrm{X}$ \\
\hline $10 \mathrm{~A}$ & 290,08829 & {$\left[\mathrm{C}_{11} \mathrm{H}_{17} \mathrm{NO}_{8}-\mathrm{H}\right]^{-}$} & D-glicose-6-[(2S)-5-oxopirrolidina-2-carboxilato] & $\mathrm{X}$ & $\mathrm{X}$ & $\mathrm{X}$ & $\mathrm{X}$ \\
\hline $11 \mathrm{~A}$ & 293,05175 & {$\left[\mathrm{C}_{17} \mathrm{H}_{26} \mathrm{O}_{4}-\mathrm{H}\right]^{-}$} & Gingerol & & & $\mathrm{X}$ & $\mathrm{X}$ \\
\hline $12 \mathrm{~A}$ & 308,09888 & {$\left[\mathrm{C}_{11} \mathrm{H}_{19} \mathrm{NO}_{9}-\mathrm{H}\right]^{-}$} & Ácido 9-O-acetilneuramínico & $\mathrm{X}$ & $\mathrm{X}$ & & \\
\hline $13 \mathrm{~A}$ & 347,18663 & {$\left[\mathrm{C}_{20} \mathrm{H}_{28} \mathrm{O}_{5}-\mathrm{H}\right]^{-}$} & Isocohumulona & $\mathrm{X}$ & $\mathrm{X}$ & $\mathrm{X}$ & $\mathrm{X}$ \\
\hline $14 \mathrm{~A}$ & 361,20226 & {$\left[\mathrm{C}_{21} \mathrm{H}_{30} \mathrm{O}_{5}-\mathrm{H}\right]^{-}$} & (+)-cis-isohumulona ou isoadhumulona ou humulona & $\mathrm{X}$ & $\mathrm{X}$ & $\mathrm{X}$ & $\mathrm{X}$ \\
\hline $2 \mathrm{~B}$ & 365,25565 & {$\left[\mathrm{C}_{19} \mathrm{H}_{38} \mathrm{O}_{4}+\mathrm{Cl}\right]^{-}$} & Ésteres & & & $\mathrm{X}$ & $\mathrm{X}$ \\
\hline $15 \mathrm{~A}$ & 377,08585 & {$\left[\mathrm{C}_{12} \mathrm{H}_{22} \mathrm{O}_{11}+\mathrm{Cl}\right]^{-}$} & Maltose & $\mathrm{X}$ & $\mathrm{X}$ & $\mathrm{X}$ & $\mathrm{X}$ \\
\hline $16 \mathrm{~A}$ & 379,08895 & {$\left[\mathrm{C}_{12} \mathrm{H}_{22} \mathrm{O}_{11}+\mathrm{Cl}\right]^{-}$} & Maltose & $\mathrm{X}$ & $\mathrm{X}$ & $\mathrm{X}$ & $\mathrm{X}$ \\
\hline 3B & 393,27145 & {$\left[\mathrm{C}_{21} \mathrm{H}_{42} \mathrm{O}_{4}+\mathrm{Cl}\right]^{-}$} & Ésteres & & & $\mathrm{X}$ & $\mathrm{X}$ \\
\hline $17 \mathrm{~A}$ & 422,22711 & {$\left[\mathrm{C}_{29} \mathrm{H}_{44} \mathrm{O}_{2}-\mathrm{H}\right]^{-}$} & Fenil éster & & & $\mathrm{X}$ & $\mathrm{X}$ \\
\hline $18 \mathrm{~A}$ & 429,12533 & {$\left[\mathrm{C}_{15} \mathrm{H}_{26} \mathrm{O}_{14}-\mathrm{H}\right]^{-}$} & Ácido 2-O-[ $\alpha$-D-manosil- (1->2- $\alpha$-D-Glucosil]-D-glicérico & $\mathrm{X}$ & $\mathrm{X}$ & & \\
\hline 19A & 449,17013 & {$\left[\mathrm{C}_{26} \mathrm{H}_{22} \mathrm{~N}_{6} \mathrm{O}_{2}-\mathrm{H}\right]^{-}$} & Piridinas & $\mathrm{X}$ & $\mathrm{X}$ & & \\
\hline 4B & 465,33171 & {$\left[\mathrm{C}_{31} \mathrm{H}_{45} \mathrm{O}_{3}-\mathrm{H}\right]^{-}$} & Vitamina K epoxidada & & & $\mathrm{X}$ & $\mathrm{X}$ \\
\hline $20 \mathrm{~A}$ & 470,15193 & 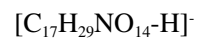 & $\alpha-N e u 5 A c-(2->3)-D-G a l$ & $\mathrm{X}$ & $\mathrm{X}$ & $\mathrm{X}$ & $\mathrm{X}$ \\
\hline $21 \mathrm{~A}$ & 539,13904 & {$\left[\mathrm{C}_{18} \mathrm{H}_{32} \mathrm{O}_{16}+\mathrm{Cl}\right]^{-}$} & Maltotriose & $\mathrm{X}$ & $\mathrm{X}$ & $\mathrm{X}$ & $\mathrm{X}$ \\
\hline $22 \mathrm{~A}$ & 541,13609 & {$\left[\mathrm{C}_{18} \mathrm{H}_{32} \mathrm{O}_{16}+\mathrm{Cl}\right]^{-}$} & Maltotriose & $\mathrm{X}$ & $\mathrm{X}$ & $\mathrm{X}$ & $\mathrm{X}$ \\
\hline $23 \mathrm{~A}$ & 631,50803 & {$\left[\mathrm{C}_{37} \mathrm{H}_{72} \mathrm{O}_{5}+\mathrm{Cl}\right]^{-}$} & Estearato de 2-hidroxi-3-(palmitoiloxi)propil & $\mathrm{X}$ & $\mathrm{X}$ & & \\
\hline $24 \mathrm{~A}$ & 653,30212 & {$\left[\mathrm{C}_{26} \mathrm{H}_{42} \mathrm{~N}_{10} \mathrm{O}_{10}-\mathrm{H}\right]^{-}$} & Peptídeos & $\mathrm{X}$ & $\mathrm{X}$ & & \\
\hline $25 \mathrm{~A}$ & 659,53465 & {$\left[\mathrm{C}_{39} \mathrm{H}_{76} \mathrm{O}_{56}+\mathrm{Cl}\right]^{-}$} & 1,2-Distearato de (S)glicerilo & $\mathrm{X}$ & $\mathrm{X}$ & & \\
\hline
\end{tabular}


Tabela 5. Modelos aplicados às técnicas analíticas de infravermelho médio (IVM) e próximo (IVP), RMN de ${ }^{1} \mathrm{H}$, ESI(+)-MS e ESI(-)-MS segundo os parâmetros de desempenho

\begin{tabular}{lcccccccc}
\hline Propriedade & Técnica & Modelo & RMSEC & RMSEP & R $^{2} \mathrm{c}$ & $\mathrm{R}^{2} \mathrm{p}$ & LD & LQ \\
\hline Cor (EBC) & NIR & PLS/ MSC & 1,0215 & 1,7954 & 0,77 & 0,31 & 0,2034 & 0,6779 \\
Acidez Total (meq L ${ }^{-1}$ ) & RMN de ${ }^{1} \mathrm{H}$ & PLS/ airPLS & 4,9077 & 8,5213 & 0,31 & 0,14 & 0,0483 & 0,1611 \\
pH & ESI(+)FT-ICR MS & PLS & 0,061 & 0,0935 & 0,88 & 0,22 & 0,0167 & 0,0557 \\
Densidade $\left(\mathrm{g} \mathrm{mL}^{-1}\right.$ ) & MIR & SVR/ MSC & 0,0016 & 0,0016 & 0,92 & 0,87 & - & - \\
Teor Alcoólico (\%v/v) & MIR & PLS & 0,2430 & 0,3929 & 0,96 & 0,95 & 0,0991 & 0,3305 \\
Amargor (IBU) & ESI (-) FT-ICR MS & PLS & 1,3022 & 1,6008 & 0,84 & 0,61 & 0,2688 & 0,8959 \\
\hline
\end{tabular}

Para a estimativa da cor da bebida (EBC) o modelo que apresentou melhor resultado, conforme apresentado na Tabela 5, foi o PLS, pré-tratado com MSC, construídos com dados do MIR. Apesar das 5 VL utilizadas no modelo somarem $94,96 \%$ da variância explicada o grupo de teste resultou em predição com acerto de $31 \%$. Foi possível identificar que a região entre 4000 a 7500 apresenta grande relevância nas informações contidas nas VL, o que pode ser associado a presença dos sinais de ligação $\mathrm{H}-\mathrm{O}$ da água, componente majoritário da bebida, de grande intensidade, em que abrange 6880 e $5115 \mathrm{~cm}^{-1}$.

A estimativa dos valores de acidez total $\left(\mathrm{meq} \cdot \mathrm{L}^{-1}\right)$ teve como melhor resultado o modelo PLS construído com dados de RMN de ${ }^{1} \mathrm{H}$, construído com 2 VL que somam $82,11 \%$ da variância explicada. Nele foi possível observar que os sinais em 1,03 ppm (tripleto) e 3,5 ppm (quadrupleto), característicos dos hidrogênios metílicos e metilênicos do etanol, respectivamente, foram os que mais influenciaram no modelo.

O modelo PLS construído com os dados da ESI(+)FT-ICR MS, mostrou-se o mais eficaz na estimativa do $\mathrm{pH}$ das cervejas. Esse modelo foi desenvolvido com 3 VL somando 58,03\% da variância explicada. Os resultados indicam acerto de $88 \%$ no conjunto de calibração e desempenho em $22 \%$ de linearidade $\left(\mathrm{R}_{\mathrm{p}}^{2}\right)$ no conjunto de teste. Para ele foi possível observar a contribuição de diversos sinais entre 500 e $1000 \mathrm{~m} / z$, que podem ser associados a aminoácidos, proteínas e carboidratos presentes na bebida e que possuem característica ácida.

Os modelos PLS construídos com dados de MIR mostraram o melhor desempenho para estimativa do ter alcoólico das bebidas, considerando tanto os valores declarados pelos fabricantes, quanto aqueles obtidos experimentalmente, possuindo linearidade maior que $95 \%$ para os valores de teste. Nesse modelo foi possível identificar a contribuição dos sinais abaixo de $1500 \mathrm{~cm}^{-1}$, em especial, as bandas em 1050-1040 e $875-865 \mathrm{~cm}^{-1}$ que podem ser atribuídas a ligação C-O de álcool primário, característico do etanol. Além disso, observa-se contribuição em $3320 \mathrm{~cm}^{-1}$ que pode ser associada a alongamentos da ligação $\mathrm{H}-\mathrm{O}$, corroborando com as análises apresentadas anteriormente sobre os dados MIR.

Amargor teve melhor resultado em sua predição quando aplicado modelo PLS nos dados de ESI(-) FT-ICR MS, construído com 3 VL que somam 50,54\% da variância explicada, no qual destacase a contribuição dos sinal em 347,18 e $361,20 \mathrm{~m} / \mathrm{z}$, atribuídos a, respectivamente, presença de isocohumulona e (+)-cis-isohumulona ou isoadhumulona ou humulona, compostos derivados do lúpulo e que são responsáveis por conferir amargor a bebida, corroborando com as análises apresentadas anteriormente sobre os dados de massas.

Adicionalmente, a partir da construção de uma PCA com os dados de MIR e ESI(+)FT-ICR MS, também foi possível classificar as cervejas, respectivamente, quanto a presença de álcool (Figura 9.A) e o método de produção (Figura 9.B). Na Figura 9.A, nota-se a formação de dois grupos bem definidos. As amostras isentas de álcool formaram um agrupamento no quadrante negativo da $1^{\text {a }}$ Componente Principal, PC1. Enquanto o eixo PC1 >0 agrupa, majoritariamente, cervejas alcoólicas, refletindo a contribuição da região de impressão digital e das bandas associadas ao etanol (1050-1040 e 875-865 cm-1). O resultado da PCA indica boa sensibilidade do MIR na distinção das cervejas zero álcool (sem etanol) das demais.

Na Figura 9.B são apresentados os gráficos de scores das duas componentes principais da PCA construídas por ESI(+)FT-ICR MS. Nota-se a formação de dois grupos bem definidos. No qual, no quadrante positivo da $1^{\text {a }}$ Componente Principal, PC1, agruparam-se as amostras artesanais, caracterizadas pela maior presença de sinais associados a compostos nitrogenados (proteínas e aminoácidos). E, no quadrante negativo da PC1, estão agrupadas, majoritariamente, as cervejas industriais, identificadas pela menor quantidade e intensidade de sinais atribuídos a proteínas e aminoácidos.
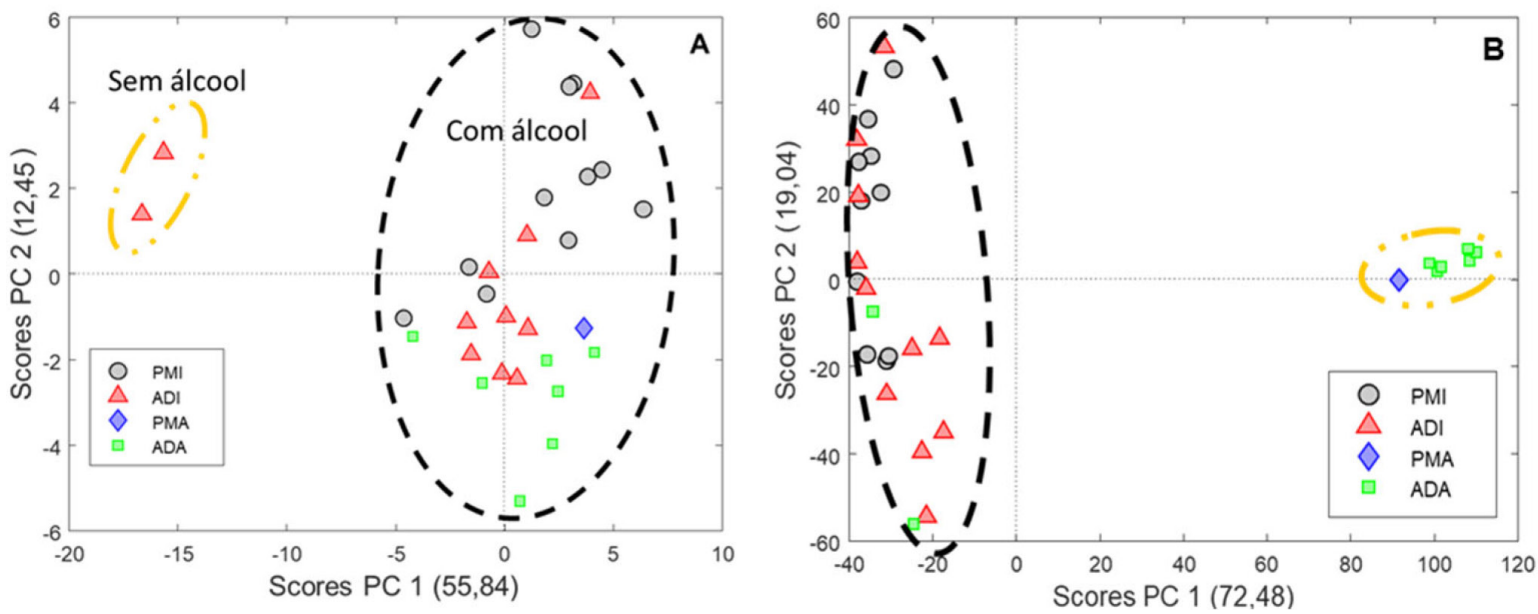

Figura 9. Gráfico de scores das duas primeiras componentes principais para MIR (A). Gráfico de scores das duas primeiras componentes principais para ESI(+)FT-ICR MS (B). Sendo: Puro Malte Industrial (PMI), Adjunto Industrial (ADI), Puro Malte Artesanal (PMA), Adjunto Artesanal (ADA) 


\section{CONCLUSÕES}

Os resultados obtidos indicam que as cervejas artesanais apresentam maior intensidade e quantidade de sinais atribuídos a aminoácidos e proteínas. Enquanto a carbonatação por adição de sacarose aumenta a consumo dos compostos nitrogenados por meio de leveduras. Com isso, as características de cervejas artesanais carbonatadas com essa técnica se tornam semelhantes às bebidas industriais. Além disso, foi observada redução na eficiência da fermentação em função do aumento na concentração $(\% \mathrm{~m} / \mathrm{m}) \mathrm{de}$ adjuntos durante a mostura, resultando em bebidas com menor teor alcoólico. Contudo, a adição de milho colabora para o aumento do amargor da cerveja, enquanto o emprego do arroz tem efeito inverso.

A aplicação de modelos quimiométricos permitiu correlacionar os espectros das amostras de cerveja com suas propriedades físicoquímicas e agrupar bebidas de acordo com sua composição e processos produtivos. Dentre as técnicas utilizadas no estudo, a MIR foi a que apresentou melhor desempenho para prever o teor alcoólico, a densidade e classificar as bebidas quanto a presença, ou não, de etanol.

Enquanto a ESI(-)FT-ICR MS apresenta potencial na predição do amargor das cervejas e na identificação do processo produtivo (artesanal e industrial). Em função da complexidade que a cerveja apresenta em sua matriz composicional, não foram obtidos resultados satisfatórios para a predição de cor, acidez total e $\mathrm{pH}$. Contudo é importante destacar que determinação experimental de amargor e teor alcoólico são as que demanda de maior tempo e uso de reagentes, indicando que os resultados obtidos podem colaborar para maior rapidez na obtenção dos dados, combinado a redução de reagentes.

\section{AGRADECIMENTOS}

Os autores agradecem aos órgãos financiadores CAPES, CNPq e FAPES pelo suporte financeiro e à Universidade Federal do Espírito Santo (UFES) por toda a estrutura disponibilizada para o desenvolvimento da presente pesquisa.

\section{REFERÊNCIAS}

1. dos Santos Bernardi, G.; Magro, J. D.; Mazutti, M. A.; Oliveira, J. V.; Di Luccio, M.; Zabot, G. L.; Tres, M. V; In Engineering Tools in the Beverage Industry; Grumezescu, A. M., Holban, A. M., eds.; Elsevier: Amsterdam 2019; pp. 405-434.

2. BRASIL; Anuário da Cerveja 2020; 2021.

3. Revista Beer Art; Brasil chega a mil fábricas de cerveja, disponível em https://revistabeerart.com/news/cervejarias-brasil-numeros, acessada em janeiro 2022.

4. Lapolli, C.; Mercado da cerveja 2018; Brasília, 2019.

5. ABRACERVA; Estatuto da associação brasileira das microvervejarias ABRACERVA, disponível em https://abracerva.com.br/sobre/estatuto/, acessado em janeiro 2022.

6. Brewers Association; Craft brewer definition, disponível em https:// www.brewersassociation.org/statistics-and-data/craft-brewer-definition/, acessada em janeiro 2022.

7. Morado, R.; Larousse da Cerveja; Editora Alaúde: São Paulo, 2017.

8. Meussdoerffer, F. G.; In Handbook of Brewing; Eßlinger, H. M., ed.; Wiley-VCH Verlag GmbH \& Co. KGaA: Weinheim, Germany, 2009; pp. $1-42$.

9. Karabín, M.; Hudcová, T.; Jelínek, L.; Dostálek, P.; Compr. Rev. Food Sci. Food Saf. 2016, 15, 542.

10. Kunze, W.; Technology, Brewing and Malting, $3^{\text {rd }}$ ed., VLB Berlin: Berlin, 2004.

11. Hughes, E. D. B. P. S.; Beer: Quality, Safety and Nutritional Aspects; The Royal Society of Chemistry: Cambridge, 2001.
12. Morales-Toyo, M.; Revista de Química PUCP 2018, 32, 4.

13. Aquilani, B.; Laureti, T.; Poponi, S.; Secondi, L.; Food Quality and Preference 2015, 41, 214.

14. Biancolillo, A.; Bucci, R.; Magrì, A. L.; Magrì, A. D.; Marini, F.; Anal. Chim. Acta 2014, 820, 23.

15. Giovenzana, V.; Beghi, R.; Guidetti, R.; J. Food Eng. 2014, 142, 80.

16. Grassi, S.; Amigo, J. M.; Lyndgaard, C. B.; Foschino, R.; Casiraghi, E.; Food Chem. 2014, 155, 279.

17. Mignani, A. G.; Ciaccheri, L.; Mencaglia, A. A.; Ottevaere, H.; Báca, E. E. S.; Thienpont, H.; Sens. Actuators, B 2013, 179, 140.

18. Fernández Pierna, J. A.; Duponchel, L.; Ruckebusch, C.; Bertrand, D.; Baeten, V.; Dardenne, P.; Chemom. Intell. Lab. Syst. 2012, 113, 2.

19. Polshin, E.; Aernouts, B.; Saeys, W.; Delvaux, F.; Delvaux, F. R.; Saison, D.; Hertog, M.; Nicolaï, B. M.; Lammertyn, J.; J. Food Eng. 2011, 106, 188.

20. Ghasemi-Varnamkhasti, M.; Forina, M.; Computers and Electronics in Agriculture 2014, 100, 34.

21. Duarte, I. F.; Barros, A.; Almeida, C.; Spraul, M.; Gil, A. M.; J. Agric. Food Chem. 2004, 52, 1031.

22. Pinto, M.; Coelho, E.; Nunes, A.; Brandão, T.; Coimbra, M. A.; Carbohydr. Polym. 2015, 116, 215.

23. Almeida, C.; Duarte, I. F.; Barros, A.; Rodrigues, J.; Spraul, M.; Gil, A. M.; J. Agric. Food Chem. 2006, 54, 700.

24. Duarte, I. F.; Godejohann, M.; Braumann, U.; Spraul, M.; Gil, A. M.; J. Agric. Food Chem. 2003, 51, 4847.

25. Duarte, I.; Barros, A.; Belton, P. S.; Righelato, R.; Spraul, M.; Humpfer, E.; Gil, A. M.; J. Agric. Food Chem. 2002, 50, 2475.

26. Rodrigues, J. E. A.; Erny, G. L.; Barros, A. S.; Esteves, V. I.; Brandão, T.; Ferreira, A. A.; Cabrita, E.; Gil, A. M.; Anal. Chim. Acta 2010, 674, 166.

27. Kuballa, T.; Brunner, T. S.; Thongpanchang, T.; Walch, S. G.; Lachenmeier, D. W.; Current Opinion in Food Science 2018, 19, 57.

28. Petersen, B. O.; Nilsson, M.; Bøjstrup, M.; Hindsgaul, O.; Meier, S.; Food Chem. 2014, 150, 65.

29. Rodrigues, J. A.; Barros, A. S.; Carvalho, B.; Brandão, T.; Gil, A. M.; Anal. Chim. Acta 2011, 702, 178.

30. da Silva, L. A.; Flumignan, D. L.; Tininis, A. G.; Pezza, H. R.; Pezza, L.; Food Chem. 2019, 272, 488.

31. Khatib, A.; Wilson, E. G.; Kim, H. K.; Lefeber, A. W. M.; Erkelens, C.; Choi, Y. H.; Verpoorte, R.; Anal. Chim. Acta 2006, 559, 264.

32. Andrés-Iglesias, C.; Blanco, C. A.; Blanco, J.; Montero, O.; Food Chem. 2014, 157, 205.

33. Araújo, A. S.; da Rocha, L. L.; Tomazela, D. M.; Sawaya, A. C. H. F.; Almeida, R. R.; Catharino, R. R.; Eberlin, M. N.; The Analyst 2005, 130, 884.

34. Pereira, H. V.; Amador, V. S.; Sena, M. M.; Augusti, R.; Piccin, E.; Anal. Chim. Acta 2016, 940, 104.

35. Martínez-Huélamo, M.; Jáuregui, O.; Estruch, R.; Lamuela-Raventós, R.; Chiva-Blanch, G.; Vallverdú-Queralt, A.; Quifer-Rada, P.; Food Chem. 2014, 169, 336.

36. Giovenzana, V.; Beghi, R.; Guidetti, R.; J. Food Eng. 2014, 142, 80.

37. BEERSMITH, version 2.3.12, 2012.

38. Dorval M. Coelho Neto, Cristina M. S. Sad, Betina P. Oliveira, Eustáquio V. R. Castro, Wanderson Romão, Paulo R. Filgueiras, V. L. J.; $19^{\circ}$ Encontro Nacional de Química Analítica e $7^{\circ}$ Congresso Iberoamericano de Química Analítica, Caldas Novas, Brasil, 2018.

39. Brasil. Ministério da Agricultura Pecuária e Abastecimento; Manual Operacional de Bebidas e Vinagres; Brasília, 2008.

40. Philpott, J.; Taylor, D. M.; Williams, D. R.; J. Am. Soc. Brew. Chem. 1997, 55, 103.

41. Analytica-EBC; Section 9 Beer. Method 9.6 Colour of Beer: Spectrophotometric Method; Bruxelas, 2010.

42. MATLAB; version 8.1.0.604 (R2013a); The MathWorks Inc.: Natick, Massachusetts, 2013. 
43. Kennard, R. W.; Stone, L. A.; Technometrics 1969, 11, 137.

44. Savorani, F.; Tomasi, G.; Engelsen, S. B.; J. Magn. Reson. 2010, 202, 190.

45. Tomasi, G.; Savorani, F.; Engelsen, S. B.; J. Chromatogr. A 2011, 1218, 7832.

46. Zhang, Z.-M.; Chen, S.; Liang, Y.-Z.; The Analyst 2010, 135, 1138.

47. Boyce, M. S.; Vernier, P. R.; Nielsen, S. E.; Schmiegelow, F. K. A.; Ecol. Modell. 2002, 157, 281.

48. Smola, A. J.; Schölkopf, B.; Statistics and Computing 2004, 14, 199.

49. Farhat, N. H.; IEEE Expert 1992, 7, 63.

50. Rosa, N. A.; Afonso, J. C.; Quim. Nova Esc. 2015, 37, 98.

51. Goiana, M. L.; Pinto, L. I. F.; Zambelli, R. A.; Miranda, K. W. E.; Pontes, D. F.; XXV Congresso Brasileiro de Ciência e Tecnologia de Alimentos 2016, 55, 1.

52. Brasil. Casa Civil; Decreto $N^{o} 6.871$ de 04 de junho de 2009; Brasil, 2009.

53. Universidade de São Paulo; Tabela Brasileira de Composição de Alimentos, disponível em http://www.tbca.net.br/index.html, acessada em janeiro 2022.

54. Ferreira, I. M.; Guido, L. F.; Fermentation 2018, 4, 23.

55. Mannina, L.; Marini, F.; Antiochia, R.; Cesa, S.; Magrì, A.; Capitani, D.; Sobolev, A. P.; Electrophoresis 2016, 37, 2710.

56. Odai, H.; Shindo, K.; Odagawa, A.; Mochizuki, J.; Hamada, M.; Takeuchi, T.; The Journal of Antibiotics 1994, 47, 939.

57. Shahidi Bonjar, G. H.; Fooladi, M. H.; Mahdavi, M. J.; Shahghasi, A.; Biotechnology (Faisalabad) 2004, 3, 126.
58. Matsui, R.; Seto, K.; Sato, Y.; Suzuki, T.; Nakazaki, A.; Kobayashi, S.; Angew. Chem., Int. Ed. 2011, 50, 680.

59. Kohno, J.; Nishio, M.; Sakurai, M.; Kawano, K.; Hiramatsu, H.; Kameda, N.; Kishi, N.; Yamashita, T.; Okuda, T.; Komatsubara, S.; Tetrahedron 1999, 55, 7771.

60. Bettiol, W.; Augusto, M.; Morandi, B.; Vegette, Z.; Trazilbo, P.; De, J.; Élida, P. J.; Corrêa, B.; Bittencourt, A.; Cleusa, M.; Lucon, M. M.; de Cássia, J.; Costa, B.; Bezerra, J. L.; Documentos 88: Produtos Comerciais à Base de Agentes de Biocontrole de Doenças de Plantas; Jaguariúna, 2012.

61. Bressan, W.; Edson Fontes Figueiredo, J.; Comunicado Técnico 65: Controle de Fusarium moniliforme em sementes de milho por actinomicetos; Sete Lagoas, 2003.

62. He, Y.; Dong, J.; Yin, H.; Zhao, Y.; Chen, R.; Wan, X.; Chen, P.; Hou, X.; Liu, J.; Chen, L.; J. Inst. Brew. 2014, 120, 157.

63. Herrera-Gamboa, J. G.; López-Alvarado, C. B.; Pérez-Ortega, E.; Damas-Buenrostro, L. C.; Cabada-Amaya, J. C.; Pereyra-Alférez, B.; J. Cereal Sci. 2018, 80, 150.

64. Cheiran, K. P.; Raimundo, V. P.; Manfroi, V.; Anzanello, M. J.; Kahmann, A.; Rodrigues, E.; Frazzon, J.; Food Chem. 2019, 286, 113.

65. Kondo, K.; BioFactors 2008, 22, 303.

66. CropLife International; Implementación del Manejo Integrado de Malezas para los Cultivos Tolerantes a Herbicidas, Brussels, 2012. 